\title{
Estudio floRístico de LA MICROCUENCA DEL CAÑóN DE LOS CHIVOS, SAN LUIS POTOSÍ, MÉXICO
}

\author{
FLORISTIC STUDY OF THE MICROBASIN OF THE LOS ChIVOS CANYON, \\ SAN LUIS POTOSI, MEXICO
}

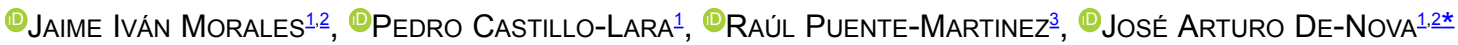 \\ ${ }^{1}$ Instituto de Investigación de Zonas Desérticas, Universidad Autónoma de San Luis Potosí, San Luis Potosí, S.L.P., México. \\ ${ }^{2}$ Facultad de Agronomía y Veterinaria, Universidad Autónoma de San Luis Potosí, Soledad de Graciano Sánchez, S.L.P., México. \\ ${ }^{3}$ Desert Botanical Garden, Phoenix, AZ, EE.UU.
}

Autor para la correspondencia: arturo.denova@gmail.com

\section{Resumen}

Antecedentes: Los ecosistemas de montaña resguardan una alta biodiversidad. La microcuenca del Cañón de Los Chivos es un eslabón importante para la biodiversidad de la Sierra Madre Oriental y un refugio para los ecosistemas de montaña de la región.

Pregunta: ¿Cuál es la composición y riqueza de plantas vasculares de la microcuenca del Cañón de Los Chivos, formas de crecimiento, distribución por tipo de vegetación, endemismo y estado de conservación?

Sitio de estudio y fechas: Microcuenca del Cañón de Los Chivos, sierra de Álvarez, San Luis Potosí, México, febrero de 2016 a junio de 2019.

Métodos: Se realizaron recolectas botánicas por tipo de vegetación, en un gradiente de 1,437 a 2,702 m snm. Se tomaron datos sobre el hábitat, fenología y forma de crecimiento.

Resultados: Se registraron 104 familias, 327 géneros y 545 especies de plantas vasculares. Las familias más ricas son Asteraceae, Fabaceae, Orchidaceae, Poaceae y Cactaceae. El área incluye 181 especies endémicas de México y 24 de San Luis Potosí y regiones adyacentes. Once especies se enlistan en la NOM-059-SEMARNAT (SEMARNAT 2010), 84 en la IUCN (seis en una categoría amenazada) y 45 en la CITES. Seis especies representan nuevos registros para el estado.

Conclusiones: La riqueza del área es relativamente mayor que en otras áreas con ecosistemas de montaña de la región. Su estado de conservación, así como la conexión con áreas naturales protegidas, endemismo y proporción de especies en categoría de riesgo, resaltan su importancia como área prioritaria para la conservación de estos ecosistemas en México.

Palabras clave: Ecosistemas de montaña, endemismo, especies en riesgo, florística, vegetación.

\begin{abstract}
Background: Mountain ecosystems shelter high levels of biodiversity. The microbasin of the Los Chivos Canyon is an important source of biodiversity of the Sierra Madre Oriental, and a refuge for mountain ecosystems in the region.

Question: What is the composition and richness of vascular plants in the microbasin of the Los Chivos Canyon, growth forms, distribution of vegetation types, endemics, and conservation status.

Study site and research period: The microbasin of the Los Chivos Canyon, Alvarez mountain range, San Luis Potosi, Mexico, between February 2016 and June 2019.

Methods: Botanical collections were carried out in each vegetation type, along a gradient from 1,437 to 2,702 m asl. Data for habitat, phenology, and growth form were recorded.

Results: 104 families, 327 genera, and 545 species of vascular plants were recorded. The richest families are Asteraceae, Fabaceae, Orchidaceae, Poaceae, and Cactaceae. The area includes 181 species endemic to Mexico and 24 to San Luis Potosí and adjacent regions. Eleven species are included in the NOM-059-SEMARNAT-201045, 84 in the IUCN Redlist (six in a threatened category), and 45 in the CITES. There are six new records for the state.

Conclusions: The species richness in the area is relatively higher than other areas having mountain ecosystems in the region. Its conservation status, as well as its proximity to natural protected areas, its endemism, and the proportion of species with some risk category, highlight the relevance of the area as a priority for the conservation of this ecosystems in Mexico.
\end{abstract}

Keywords: Mountain ecosystems, endemism, endangered species, floristics, vegetation. 
Los ecosistemas de montaña resguardan una alta diversidad biológica, propiciada por factores fisiográficos, geológicos, hidrológicos, climáticos y edáficos, los cuales, tras su interacción, generan ambientes heterogéneos, mismos que se ven favorecidos por la compresión climática en distancias relativamente cortas a través del gradiente altitudinal (Körner \& Spehn et al. 2002, Spehn et al. 2010). Las montañas han sido llamadas "torres de agua", ya que mejoran la calidad de vida para las personas a través de procesos que se desarrollan a través de la interacción de los componentes bióticos y abióticos, como es la aportación de agua limpia entre muchos otros servicios necesarios en el régimen biológico (Viviroli et al. 2003, Egan \& Price 2014).

En México, una las unidades fisiográficas de mayor biodiversidad y riqueza es la provincia Sierra Madre Oriental (SMOr), que resguarda poco más de 8,500 especies de plantas vasculares nativas (Luna et al. 2004, SalinasRodríguez 2015, Salinas-Rodríguez et al. 2017), de las cuales, en los últimos 30 años, se han descrito cerca de 400 especies nuevas (Rzedowski 2015). Dentro de esta provincia existen relictos bien conservados de ecosistemas naturales complejos, propios de la región, como es el caso de la Microcuenca del Cañón de los Chivos (MCC) (Figura 1). Se localiza en la vertiente este de la sierra de Álvarez (INEGI 2001) y forma parte de una pequeña porción del Área de Protección de Flora y Fauna Sierra de Álvarez, de acuerdo con Torres \& Sierra (2003).
Particularmente en México, las geoformas conocidas como cañones resaltan por su complejidad, tipos de vegetación y alta riqueza de especies asociada. Algunos ejemplos de ello son el Cañón del Espinazo del Diablo en San Luis Potosí, el Cañón del Zopilote en Guerrero, el Cañón del Sumidero en Chiapas y el Cañón de Iturbide en Nuevo León (ÁvilaSánchez et al. 2010, Salinas-Rodríguez et al. 2013, Espinosa-Jiménez et al. 2014, Castillo-Gómez 2015).

En toda la sierra de Álvarez, desde que los europeos llegaron a esta región, se ha hecho un uso irracional de sus recursos, principalmente por la extracción de madera para construcción y combustible por parte de compañías como "The Alvarez Land and Timber Company", que tuvo como objetivo proveer de leña y madera a la Compañía Metalúrgica Mexicana desde finales de la década de 1880 hasta la de 1940 (Martínez 2003, Palacios 2008), así como la extracción de roca caliza para la elaboración de cal hidratada, inicialmente por varias caleras artesanales pequeñas y más recientemente, por una gran industria como lo es CAL QUÍMICA MEXICANA, S.A. de C.V., la cual desde finales de la década de 1970 explota la roca caliza (Castillo-Lara 2003, 2007, Enríquez-Salaices-Valdez 2016). El objetivo de este trabajo fue elaborar un inventario de plantas vasculares para la MCC. Se analiza su riqueza por grupos taxonómicos, formas de crecimiento, nivel de endemismo y vulnerabilidad.

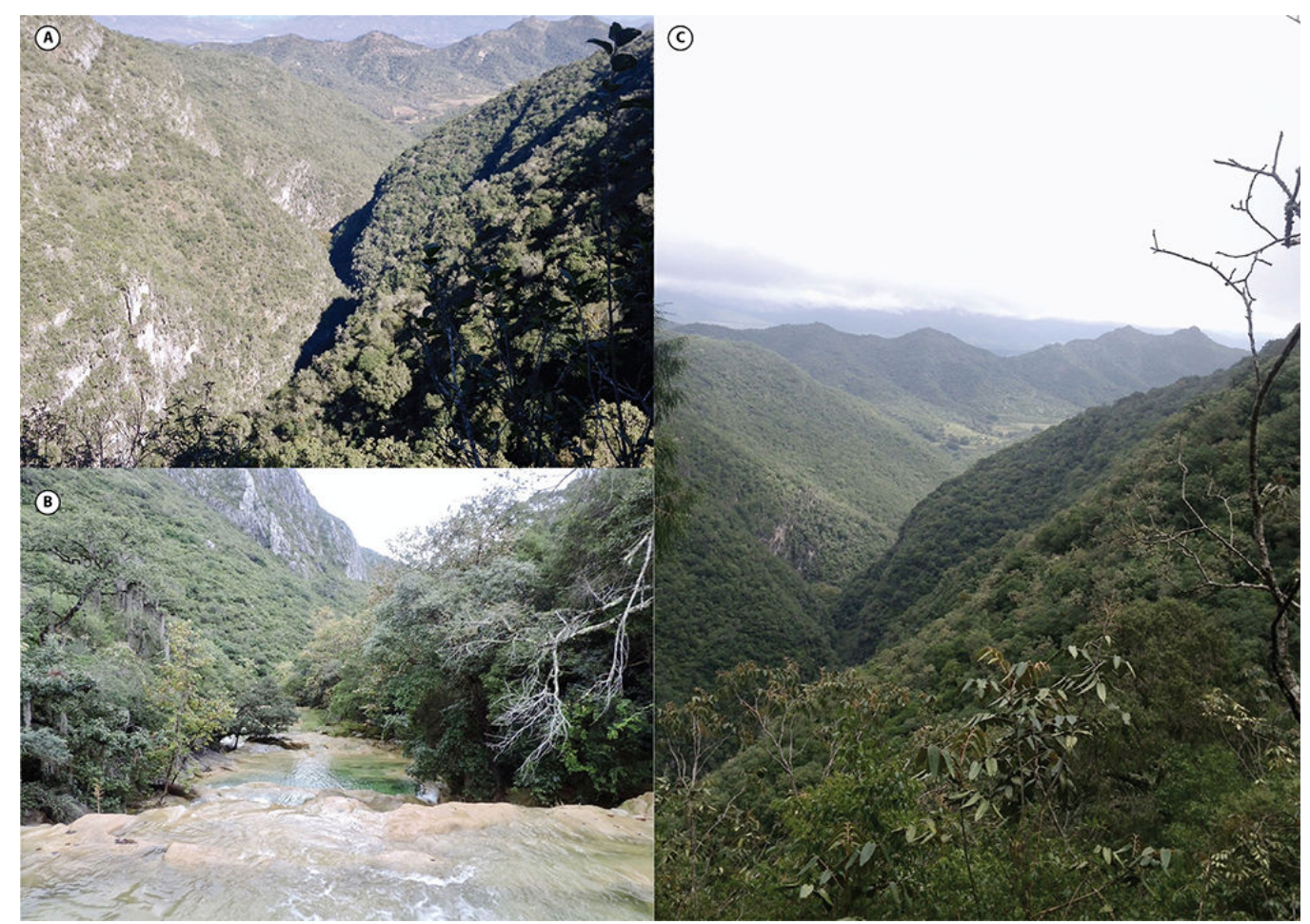

Figura 1. Paisaje de la MCC, San Luis Potosí, México. A) Ladera alta, dirección oeste-este; B) Fondo de cañón, dirección oeste-este; C) Ladera alta, dirección oeste-este, temporada funcional húmeda. Fotos J.I. Morales de la T. 


\section{Materiales y métodos}

Zona de estudio. La MCC pertenece a la Región Hidrológica del Río Panuco, dentro de la sección más alta de la Subcuenca del Río Verde (Figura 2). En la zona se presentan siete arroyos de los cuales dos son principales, arroyo Merlín y arroyo Charco Oscuro, los cuales al unirse con el arroyo La Salitrera forma el río Santa Catarina (INEGI 2001). La delimitación de la MCC se realizó a través del simulador de flujos de cuencas hidrográficas SIATL de INEGI (INEGI 2013). La MCC se localiza en los municipios de Villa de Zaragoza y San Nicolás Tolentino en el centro del estado de San Luis Potosí. Comprende una superficie total de 6,518 hectáreas y se ubica entre las coordenadas UTM: 334,819 E, 2,438,406 N, noroeste; 331,810 E, 2,437,139 N, noreste; 340,159 E, 2,417,903 N, suroeste; 344,593 E, 2,431,579 N, sureste. (INEGI 2001, 2013). La MCC se sitúa dentro de la provincia fisiográfica SMOr y subprovincia Carso Huasteco (Cervantes-Zamora et al. 1990), caracterizada localmente por sus abruptas laderas, con pendientes mayores al $20 \%$, y un gradiente altitudinal que va de los 1,437 a $2,702 \mathrm{~m}$ snm (INEGI 2013). La composición lítica en la MCC es compleja como resultado de distintos fenómenos geológicos (Carrasco 1970, Carrillo-Bravo 1971, Sánchez \& Soberanes 2001, López-Doncel 2002). Su porción norte y centro incluye rocas calizas; en una pequeña fracción al noroeste, roca lutita, mezclado en bajas porciones con roca caliza; y en la porción sur toba riolítica-ignimbrita (Sánchez \& Soberanes 2001). El suelo dominante es el litosol, con puntuales asociaciones de litosol con regosol eútrico al suroeste de la microcuenca y litosol con rendzina al sureste; al norte existe una pequeña porción de suelo luvisol órtico (INEGI 2001).

Según la clasificación de Köppen, modificado por García (1981), el clima del área pertenece al grupo $\mathrm{BS}_{1} \mathrm{kw}$, con un clima semi-seco templado con lluvias en verano (INEGI 1987, García-Sánchez \& Aguirre 2011). En la región en donde se localiza la MCC, la vegetación es compuesta en mayor proporción por encinares; sin embargo, existen otros tipos de vegetación en el área, mismos que corresponden a bosque de encino-pino, bosque de galería, matorral submontano y pastizal inducido (INEGI 2001).

Trabajo de campo y gabinete. El trabajo de campo consistió en recolectas botánicas realizadas de febrero del 2016 a junio de 2019. Se efectuó un muestro sistematizado a través de 25 transectos (Figura 2) estratificados por tipos de vegetación de acuerdo con las clasificaciones de Flores et al. (1971) y Rzedowski (1978), cada uno con una longitud promedio de $400 \mathrm{~m}$ en dirección paralela a la pendiente. Cada transecto se repitió tres veces, con base en la temporada funcional del año (húmeda, seca y fría), principalmente durante el periodo comprendido entre marzo del 2018 a julio del 2019. Se recolectaron aquellas especies
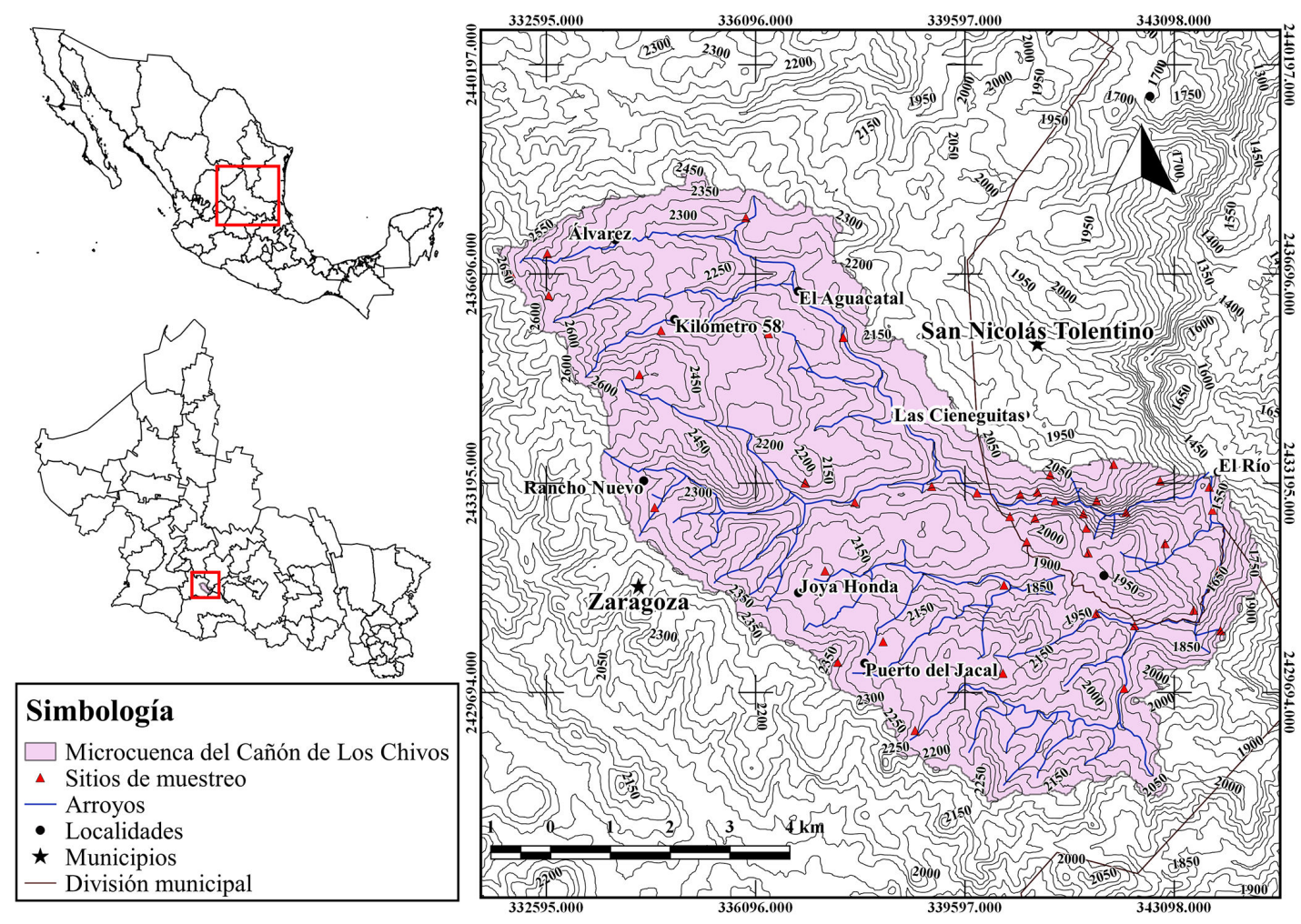

Figura 2. Localización de la MCC, San Luis Potosí, México y la ubicación de los puntos de muestreo. 
vegetales con estructuras reproductiva (flor y/o fruto) de los estratos herbáceo, arbustivo y arbóreo, en los diferentes tipos de vegetación. Se recolectaron al menos cinco duplicados por espécimen, pero para especies poco abundantes, se recolectó un ejemplar. Para las especies en riesgo únicamente se tomó registro fotográfico. Con el fin de ilustrar la apariencia estructural de cada especie se asignaron formas de crecimiento generales, de acuerdo con conceptos definidos en el glosario botánico de Font Quer (1953) como árboles, arbustos, hierbas, epífitas y trepadoras, además de casos específicos de hábitos nutricionales como lo son las parásitas y saprófitas.

Para la identificación taxonómica se revisaron tratamientos taxonómicos, descripciones de especies y guías (Salazar 1990, Herrera 2001, Espejo-Serna et al. 2002, Mickel \& Smith 2004, Borhidi 2006, Mendoza-Ruiz \& Pérez-García 2009, Martínez-Gordillo et al. 2013, Hernández-Sandoval 2019, García-Sánchez \& Aguirre 2011), además de fascículos y capítulos de Flora del Bajío y Regiones Adyacentes (Fryxell 1993, Ballard 1994, Calderón de Rzedowski \& Rzedowski 1994, CarranzaGonzález \& Madrigal 1995, García \& Koch 1995, Fernández-Nava 1996, Rzedowski \& Calderón de $\underline{\text { Rzedowski 1997, Rzedowski \& Calderón de Rzedowski }}$ 2002, Daniel \& Acosta-Castellanos 2003, Lorea-Hernández 2004, Rzedowski \& Calderón de Rzedowski 2005, PérezCalix \& Carranza-González 2005, Steinmann 2005, Calderón de Rzedowski \& Rzedowski 2006, Andrade et al. 2007, Carranza-González 2007, Carranza-González 2008, Espejo-Serna et al. 2009. Espejo-Serna et al. 2010a, 2010b, Rzedowski et al. 2011, Rzedowski \& Calderón de Rzedowski 2011, Sosa \& Valdivieso 2013, GonzálezElizondo \& González-Elizondo 2015, Martínez 2015, Rzedowski et al. 2016), de Flora Mesoamericana (Hoch et al. 2009, Stevens \& Morales 2009, Pool \& Knapp 2012), de Flora Fanerogámica del Valle de México (Calderón de Rzedowski \& Rzedowski 2005) y de Flora de Veracruz (Espejo-Serna \& López-Ferrari 2003). El procesamiento de los especímenes botánicos se realizó de acuerdo con el manual de herbario de Lot \& Chiang (1986). El secado, identificación, montaje y etiquetado de los ejemplares botánicos se realizó en el herbario Isidro Palacios (SLPM). Algunas de las especies se registraron de manera fotográfica y fueron incluidas dentro del portal Naturalista (CONABIO 2019). Adicionalmente se consultaron especímenes históricos para el área de estudio en la colección del herbario SLPM y Desert Botanical Garden (DES).

Para la clasificación de angiospermas se utilizó el sistema APG IV (스 2016) y para los helechos y afines, así como para gimnospermas el sistema de Christenhusz et al. (2011). La estandarización nomenclatural se realizó con el paquete Taxonstand v.2.1 (Cayuela et al. 2012) para R ( $\underline{\mathrm{R}}$ Development Core Team 2019), la cual se basa en ThePlantList (2010). Para el caso de taxones irresueltos se tomó de referencia el Catálogo de las plantas vasculares nativas de México (Villaseñor 2016). Se realizó una revisión exhaustiva del marco legal para conocer las especies incluidas en alguna categoría de conservación nacional en la NOM-059-ECOL-2010 (SEMARNAT 2010) o internacionales, como lo es la Convención sobre el Comercio Internacional de Especies Amenazadas de Fauna y Flora Silvestres (CITES 2019) y la lista roja de especies amenazadas de la Unión Internacional para la Conservación de la Naturaleza (IUCN 2019). Se indican las plantas restringidas al estado de San Luis Potosí y regiones adyacentes mencionadas en De-Nova et al. (2018), las especies que no son nativas de México, así como nuevos registros para el estado de acuerdo con la lista de Villaseñor (2016).

Con el fin de determinar el esfuerzo de muestreo en el área, se realizó una curva de acumulación de especies, la cual representa gráficamente la forma en que se registran nuevas especies por unidad de muestreo (Villarreal et al. 2006). Se utilizó el estimador no paramétrico Jackknife primer orden (Jack 1), el cual es una función del número de especies presentes en sólo una unidad de muestreo, mediante el software Estimates 9.1.0. (Colwell et al. 2012), con 1,000 rarefacciones y 16 extrapolaciones. Este estimador ha demostrado ser el más apropiado en términos de exactitud global (95\%), aún con un bajo número de muestras (González-Oreja et al. 2010). Para comparar la riqueza florística de la MCC y otras áreas de zonas montañosas previamente estudiadas, se calculó el índice de biodiversidad taxonómica (IB) de acuerdo con Squeo et al. (1998), definido como el número de especies entre el logaritmo natural del área en $\mathrm{km}^{2}(\mathrm{IB}=\mathrm{S} / \ln \mathrm{A})$.

\section{Resultados}

Trabajo de campo. Se realizaron un total de 871 recolectas durante 71 días desde febrero del 2016 hasta julio del 2019 , en campañas de siete días en promedio y que incluyeron todas las estaciones del año. Los ejemplares pueden ser consultados en la base de datos del herbario Isidro Palacios (SLPM 2019). Los especímenes históricos de la zona de estudio incluyen 120 ejemplares recolectados desde junio de 1998 hasta diciembre del 2015, además de un ejemplar de Edward Palmer de 1922 depositado en el herbario US. De acuerdo con el estimador Jack 1 el esfuerzo de muestreo alcanzó el $93 \%$ de la riqueza de especies esperadas para la MCC (Figura 3).

Riqueza taxonómica y formas de crecimiento. Para la MCC se registraron 545 especies de plantas vasculares, las cuales pertenecen a 327 géneros, de 104 familias y cinco linajes (Apéndice 1, Tabla 1). Se obtuvieron un total de 987 registros, de los cuales 979 cuentan con respaldo de ejemplar de herbario y ocho fueron observados y registrados 
fotográficamente en la plataforma en línea Naturalista (Apéndice 1). Las eudicotiledóneas son el grupo taxonómico mejor representado con 385 especies (70.64 \%), seguidas de las monocotiledóneas con 115 (20.10\%), y las restantes se dividen en magnolídeas con nueve $(1.65 \%)$, gimnospermas con $10(1.83 \%)$ y los helechos y afines con $26(4.77 \%)$, respectivamente (Tabla 1). Las familias más ricas son Asteraceae (37 géneros y 64 especies), Fabaceae (24 géneros y 38 especies), Orchidaceae (21 géneros y 33 especies), Poaceae (22 géneros y 29 especies) y Cactaceae (ocho géneros y 19 especies) (Tabla 2). Los géneros más diversos fueron Quercus con 17 especies, Tillandsia con nueve, Agave y Pinus con ocho, Echeveria con siete, Asclepias, Euphorbia, Salvia y Sedum con seis, y Ageratina, Panicum y Polypodium con cinco especies, respectivamente. En las figuras 4 y $\underline{5}$ se muestran algunas de las especies de la MCC. El IB dentro de la MCC es de 130.47 (spp./lnA), lo que la ubica en el segundo lugar en comparación con otras regiones estudiadas previamente en ecosistemas de montaña del estado de San Luis Potosí (Tabla 3). La forma de crecimiento dominante son las hierbas con 314 especies (57.61\%), seguidas por los arbustos con 103 especies (18.9\%), los árboles con 80 especies $(14.67 \%) \mathrm{y}$, en menor proporción las trepadoras con 31 especies $(5.68 \%)$ y las epífitas con 17 especies $(3.12 \%)$ (Tabla 4).

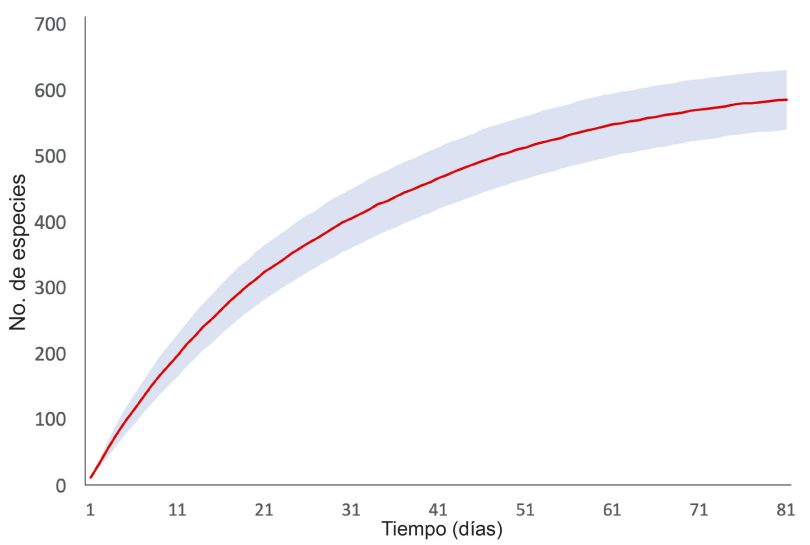

Figura 3. Curva de acumulación de especies registradas para la MCC durante el periodo de febrero del 2016 a junio del 2019.

Endemismos, especies en riesgo y nuevos registros. En la MCC se registraron 181 especies endémicas para México (118 géneros y 54 familias) y un total de 22 especies de distribución restringida al estado de San Luis Potosí y regiones adyacentes. Se registraron 117 especies dentro de la NOM-059-SEMARNAT (SEMARNAT 2010), la CITES o la IUCN (Apéndice 2), lo que representa el $21.46 \%$ de la riqueza total del área. Un total de 45 especies están protegidas por la CITES, de las cuales sólo una (Ceratozamia zaragozae) se indica en el Apéndice I (especie en mayor grado de peligro) y las otras 44 en el Apéndice II (especies que pueden estar en peligro si no se controla estrictamente su comercio). Se registró un total de 84 especies dentro de la IUCN, de las cuales una se encuentra en peligro crítico (Ceratozamia zaragozae), dos en peligro (Opuntia megarhiza y Opuntia pachyrrhiza), tres vulnerables (Cornus disciflora, Mammillaria schiedeana, Pinus greggii), dos casi amenazadas (Ferocactus histrix, Pistacia mexicana), dos de bajo riesgo dependientes de conservación (Arbutus xalapensis, Viburnum elatum) de acuerdo con la antigua categoría LR/CD (Lower risk: conservation dependant) y 74 en preocupación menor. Además, en la MCC se registraron 11 especies en alguna categoría de riesgo según la NOM-059 (SEMARNAT-2010), cuatro amenazadas (Erythrina coralloides, Litsea glaucescens, Mammillaria erythrosperma, Mammillaria schiedeana), cuatro sujetas a protección especial (Brahea moorei, Ferocactus histrix, Pinus strobiformis, Schiedeella nagellii) y tres en peligro de extinción (Ceratozamia zaragozae, Echeveria elegans, Tilia americana var. mexicana). Se registraron 17 especies no nativas de México. Además, se encontraron seis nuevos registros para el estado de San Luis Potosí, de los cuales no existía recolecta previa en herbarios ni mención en algún trabajo florístico para el estado (Corallorhiza maculata, Corallorhiza wisteriana, Funkiella tenella, Hexalectris nitida, Schiedeella crenulata y Sisyrinchium angustissimum).

Tabla 1. Número de especies de la flora vascular registradas en la MCC, San Luis Potosí, México.

\begin{tabular}{lcccc}
\hline Grupo taxonómico & Familias & Géneros & Especies & \% \\
\hline Helechos y Afines & 7 & 17 & 26 & 4.77 \\
Gimnospermas & 3 & 3 & 10 & 1.83 \\
Angiospermas & 94 & 307 & 509 & 93.39 \\
Mangnólidas & 2 & 4 & 9 & 1.65 \\
Monocotiledóneas & 15 & 67 & 115 & 20.10 \\
Eudicotiledóneas & 77 & 236 & 385 & 70.64 \\
Total & $\mathbf{1 0 4}$ & $\mathbf{3 2 7}$ & $\mathbf{5 4 5}$ & $\mathbf{1 0 0}$ \\
\hline
\end{tabular}

Vegetación. En la MCC se apreciaron fisonómicamente cinco tipos de vegetación: bosque de encino, bosque de encino-pino, bosque de galería, bosque afín a mesófilo de montaña y matorral submontano (Figura 6). Los tipos de vegetación de mayor a menor riqueza fueron el bosque de encino con 75 familias, 182 géneros y 250 especies (45.87\%), el bosque de galería con 73 familias, 170 géneros y 215 especies ( $39.45 \%$ ), el bosque de encinopino con 62 familias, 149 géneros y 211 especies (38.72\%), y el bosque afín a mesófilo de montaña con 34 familias, 
48 géneros y 51 especies (9.35\%) (Tabla 5). La forma de crecimiento más dominante en todos los tipos de vegetación fueron las hierbas, seguido por los arbustos en el bosque de encino, bosque de encino-pino, matorral submontano y por árboles en el bosque de galería y el bosque afín a mesófilo de montaña (Tabla 4). Los tipos de vegetación con mayor presencia de epífitas fueron el bosque afín a mesófilo de montaña y el matorral submontano que también tienen una mayor presencia de trepadoras.

Tabla 2. Familias con mayor riqueza de géneros y especies en la MCC, San Luis Potosí México.

\begin{tabular}{lcccc}
\hline Familia & Géneros & \% & Especies & \% \\
\hline Asteraceae & 37 & 11.31 & 64 & 11.74 \\
Fabaceae & 24 & 7.34 & 38 & 6.97 \\
Orchidaceae & 21 & 6.42 & 33 & 6.06 \\
Poaceae & 22 & 6.73 & 29 & 5.32 \\
Cactaceae & 8 & 2.45 & 19 & 3.49 \\
Fagaceae & 1 & 0.31 & 17 & 3.12 \\
Lamiaceae & 7 & 2.14 & 17 & 3.12 \\
Asparagaceae & 5 & 1.53 & 14 & 2.57 \\
Crassulaceae & 3 & 0.92 & 15 & 2.75 \\
Apocynaceae & 7 & 2.14 & 14 & 2.57 \\
Euphorbiaceae & 6 & 1.83 & 14 & 2.57 \\
Solanaceae & 7 & 2.14 & 14 & 2.76 \\
Ericaceae & 8 & 2.45 & 9 & 1.65 \\
Rubiaceae & 8 & 2.45 & 9 & 1.65 \\
Plantaginaceae & 5 & 1.53 & 6 & 1.10 \\
Total & $\mathbf{1 6 9}$ & $\mathbf{5 1 . 6 8}$ & $\mathbf{3 1 2}$ & $\mathbf{5 7 . 2 5}$ \\
\hline
\end{tabular}

\section{Discusión}

Riqueza florística. La MCC incluye al menos 545 especies de plantas superiores, lo que equivale al $10.06 \%$ de las plantas vasculares nativas del estado de San Luis Potosí que se estima en 5,413 especies (Villaseñor 2016, De-Nova 2018). Las familias más ricas en especies dentro de la MCC (Asteraceae, Fabaceae, Orchidaceae y Poaceae) resultan ser también las que se reportan como las de mayor riqueza en México (Rzedowski 1972, Villaseñor 2016, Torres-Colín et al. 2017, Sánchez-Ken 2019). Así mismo, siete de los géneros más ricos en la $\mathrm{MCC}$ coinciden con los más ricos a nivel nacional de acuerdo con Villaseñor (2016), entre los que destacan Quercus, Tillandsia, Agave y Pinus. México es considerado como centro de diversidad y origen para estos géneros o algunas de sus secciones (Gentry 1982, Farjon 1996, Valencia-A. 2004, García-Mendoza 2004, Sosa \& De-
Nova 2012, Pinzón et al. 2016, Sosa et al. 2018). Por ejemplo, la SMOr representa uno de los centros de origen y mayor riqueza específica del género Quercus (Govaerts \& Frodin 1998, Valencia-A. 2004). Se ha mencionado previamente que la riqueza y endemismo de Quercus está asociada a la combinación de varios factores, entre ellos la disponibilidad de hábitats adecuados para ser ocupados (Valencia-A. 2004). En la MCC se combinan diversas comunidades vegetales y en todas ellas el género Quercus está presente con al menos dos especies.

El valor del índice de biodiversidad taxonómica en la MCC $(\mathrm{IB}=130.47)$, sobrepasa el valor promedio $(\mathrm{IB}=108.29)$ reportado para otras regiones de ecosistemas de montaña en el estado de San Luis Potosí (Reyes-Agüero et al. 1996, García-Sánchez et al. 1999, González et al. 2007, Castillo-Gómez 2015, Torres-Colín et al. 2017, Enríquez-Salaices-Valdez 2019), y sólo se encuentra por debajo del Cañón del Espinazo del Diablo, área que incluye tipos de vegetación más complejos con afinidad cálido húmedo y subhúmedo, además de templado húmedo.

En la MCC existen especies de afinidad Neártica, pero también otros con afinidad Neotropical que se han señalado previamente con preferencia por los climas cálidos subhúmedos como son Ficus cotinifolia, Lysiloma acapulcense, Catasetum integerrimum y Arisaema macrospathum (Salazar 1990, Croat \& Carlsen 2003, Andrade et al. 2007, Carvajal 2007, Ibarra-Manríquez et al. 2012). Estas especies están presentes en el área de estudio dentro del bosque afín a mesófilo de montaña y el matorral submontano; su presencia indica condiciones ambientales y ecológicas particulares dentro de la $\mathrm{MCC}$, pues su registro en la zona representa el más continental dentro del estado. En el caso de $A$. macrospathum, su registro más continental se había mencionado para el fragmento de bosque mesófilo de Ríoverde (Salinas-Rodríguez \& Cruzado-Cortés 2011), el cual se localiza $35 \mathrm{~km}$ al este de la MCC. Por otro lado, los géneros Ficus y Catasetum no cuentan con registros para el estado fuera de la región Huasteca.

Se ha mencionado que la compleja fisiografía presente en algunos cañones incide directamente en el establecimiento de una mayor variedad de asociaciones vegetales y una alta riqueza florística (Ávila-Sánchez et al. 2010, CastilloGómez 2015). En el caso de la MCC, el contacto abrupto entre laderas con diferente exposición genera comunidades vegetales contrastantes en distancias cortas como el bosque de encino en laderas de exposición norte, con presencia de Quercus furfuracea, Q. polymorpha, Persea liebmannii, P. americana e Ilex brandegeeana y el matorral submontano en laderas de exposición sur con presencia de Juniperus flaccida, Viguiera trachyphylla, Painteria elachistophylla, Decatropis bicolor, Colubrina ehrenbergii, Bernardia albida. Estos tipos de vegetación son limitados por una delgada franja de bosque de galería en el fondo de cañón, donde la especie dominante es Platanus mexicana. 
Morales et al. / Botanical Sciences 98(4): 644-681. 2020
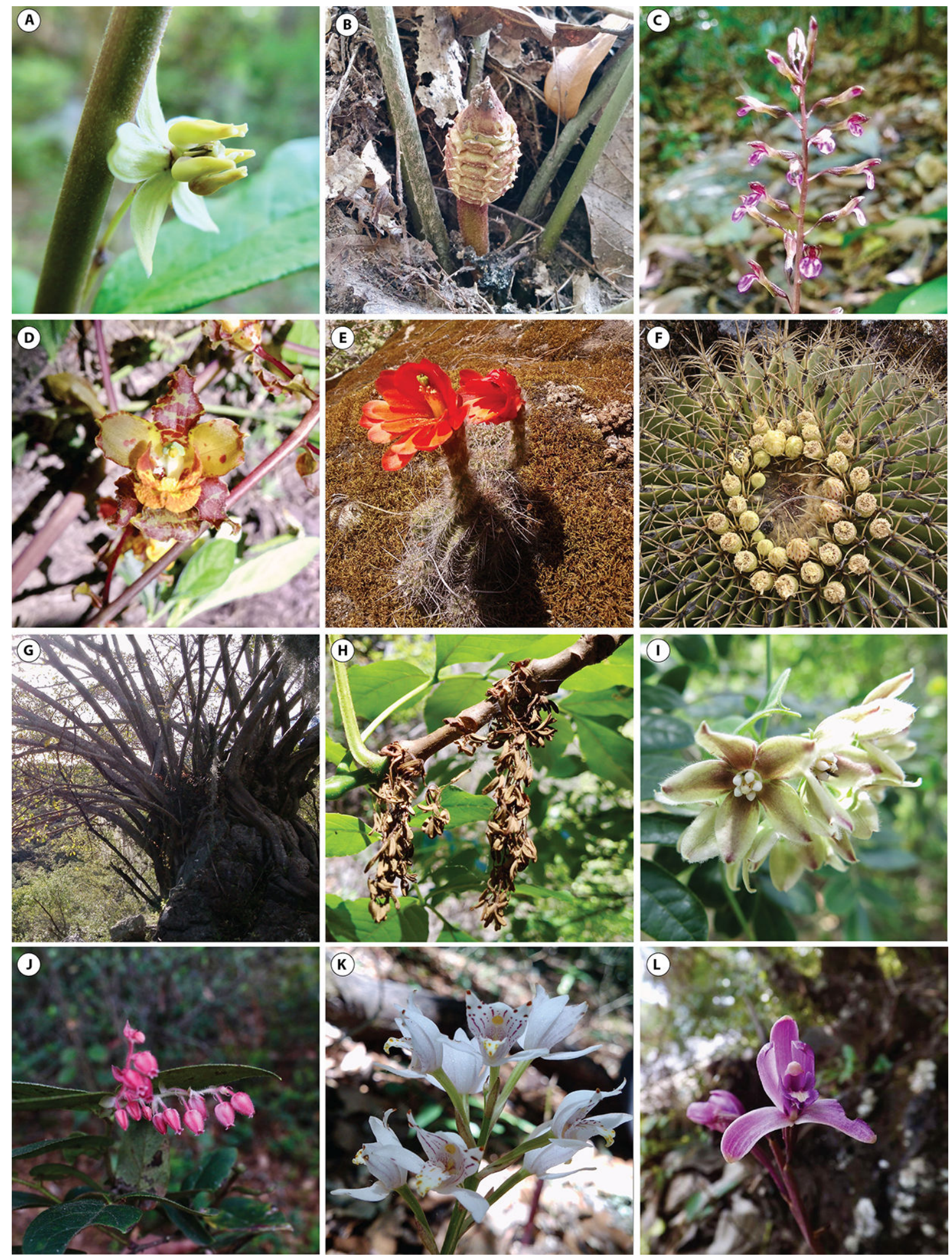

Figura 4. Algunas especies vegetales de la MCC. A) Asclepias auriculata; B) Ceratozamia zaragozae; C) Corallorhiza wisteriana; D) Cyrtopodium macrobulbon; E) Echinocereus polyacanthus var. densus; F) Ferocactus histrix; G) Ficus cotinifolia; H) Fraxinus uhdei; I) Funastrum elegans; J) Gaultheria erecta; K) Govenia purpusii; L) Hexalectris grandiflora. Fotos A, B, C, D, E, F, G, H, I: J.I. Morales de la T. Foto J: P. Castillo Lara. 
Flora de la microcuenca del Cañón de Los Chivos
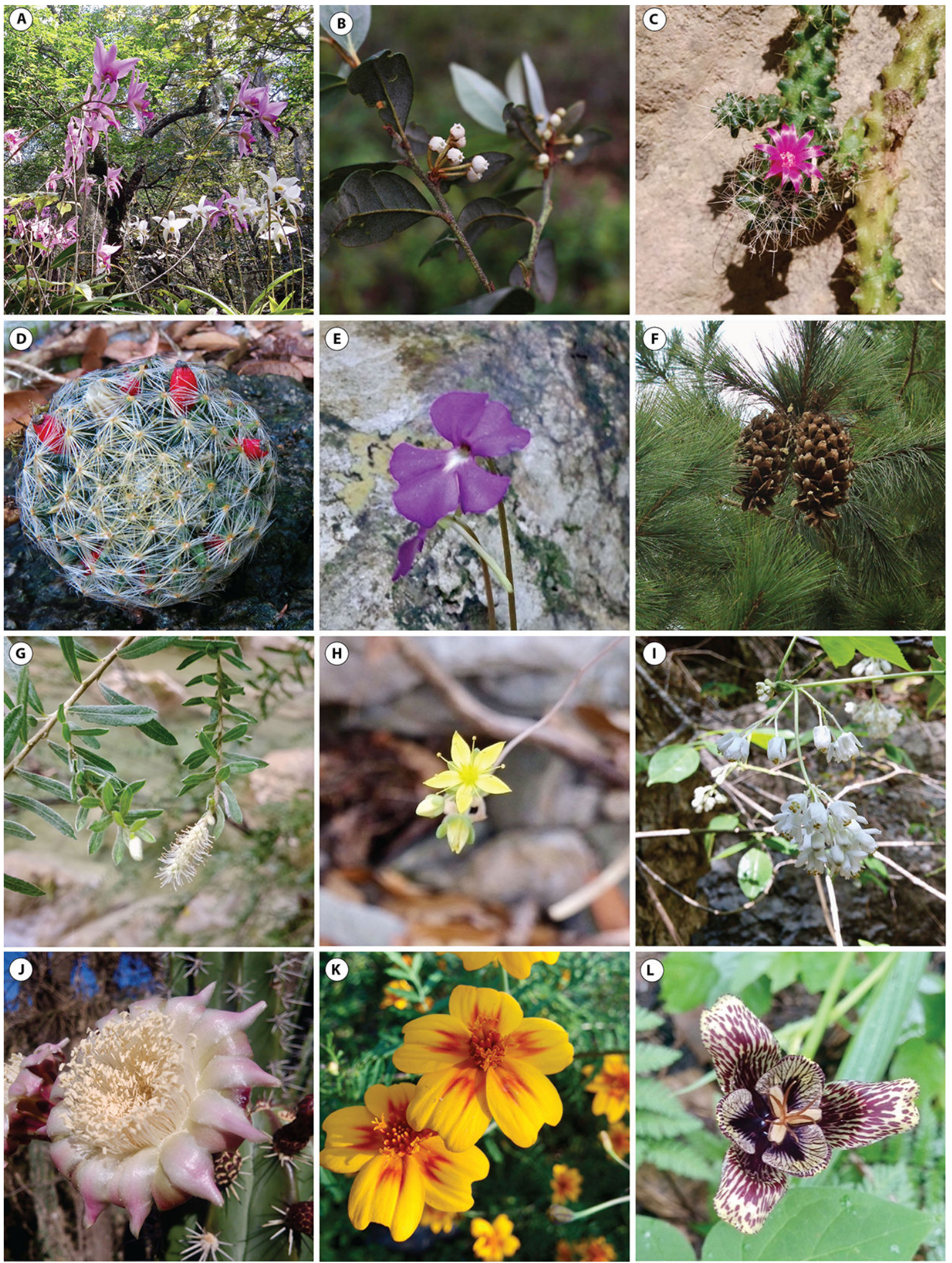

Figura 5. Algunas especies vegetales de la MCC. A) Laelia anceps; B) Lyonia squamulosa; C) Mammillaria erythrosperma; D) Mammillaria schiedeana; E) Pinguicula ehlersiae; F) Pinus strobiformis; G) Salix taxifolia; H) Sedum clausenii; I) Staphylea pringlei; J) Stenocereus queretaroensis; K) Tagetes lunulata; L) Tigridia vanhouttei. Fotos A, C, D, E, G, H, I, J, K, L J.I. Morales de la T. Foto B: P. Castillo Lara. Foto F: A.K. Gudiño Cano. 
Tabla 3. Índice de biodiversidad taxonómica IB (spp./lnA) para algunos sitios con ecosistemas de montaña en el estado de San Luis Potosí en comparación con la MCC. $\mathrm{Sc}=$ Selva baja caducifolia, $\mathrm{Ss}=$ Selva mediana subperennifolia, $\mathrm{Sp}=$ Selva mediana perennifolia, $\mathrm{Be}=\mathrm{Bosque}$ de encino, $\mathrm{Bp}=$ Bosque de pino, Bep = Bosque de encino-pino, Bpe = Bosque de pino-encino, $\mathrm{Bg}=$ Bosque de galería, Bmm = Bosque mesófilo de montaña, $\mathrm{Bm}=$ afin a Bosque mesófilo de montaña, $\mathrm{Mx}=$ Matorral xerófilo, $\mathrm{Mc}=$ Matorral crasicaule, Ms $=$ Matorral submontano, $\mathrm{Mm}=$ Matorral micrófilo, $\mathrm{Mr}=$ Matorral rosetófilo, $\mathrm{Ch}=$ Chaparral, $\mathrm{P} \tilde{n}=$ Piñonar, $\mathrm{Pz}=$ Pastizal, $\mathrm{Cd}=\mathrm{Cedral}$.

\begin{tabular}{ccccccc}
\hline Sitio de estudio & Autores & Tipo de vegetación & $\begin{array}{c}\text { Rango } \\
\text { altitudinal }\end{array}$ & $\begin{array}{c}\text { Área } \\
\left(\mathbf{k m}^{2}\right)\end{array}$ & $\begin{array}{c}\text { Riqueza } \\
\text { gamma }\end{array}$ & IB \\
\hline $\begin{array}{c}\text { Cañón del Espinazo del } \\
\text { Diablo }\end{array}$ & $\underline{\text { Castillo-Gómez 2015 }}$ & Sc, Ss, Sp, Be, Bmm, Bg, Ms & $550-1,550$ & 18 & 573 & $\mathbf{1 9 8 . 2 4}$ \\
$\begin{array}{c}\text { Cañón de los Chivos } \\
\text { Parque Nacional Potosín }\end{array}$ & Presente estudio & Be, Bep, Bg, Bm, Ms & $1,437-2,702$ & 65.18 & 545 & $\mathbf{1 3 0 . 4 7}$ \\
& $\frac{\text { Enríquez-Salaices-Valdez }}{\underline{2019}}$ & Bep, Bpe, Bg & $1,580-2,800$ & 20 & 327 & $\mathbf{1 0 9 . 1 5}$ \\
Sierra de Monte Grande & $\underline{\text { Reyes-Agüero et al. 1996 }}$ & Mm, Mr, Mc, Ch, Pñ & $2,200-2,400$ & 42.8 & 397 & $\mathbf{1 0 5 . 6 8}$ \\
Flora de Guadalcázar & Torres-Colín et al. 2017 & Be, Bp, Ms, Mx, Pz. & $1,000-2,500$ & 3,800 & 813 & $\mathbf{9 8 . 6 3}$ \\
Sierra de Catorce & González et al. 2007 & Be, Pñ, Cd, Ch, Ms, Mr, Mm & $1,400-3,060$ & 1,400 & 530 & $\mathbf{7 3 . 1 6}$ \\
Sierra de Álvarez & García-Sánchez et al. 1999 & Diferentes asociaciones de Be & $200-2,702$ & 2,276 & 332 & $\mathbf{4 2 . 9 4}$ \\
\hline
\end{tabular}

Vegetación. De los cinco tipos de vegetación presentes en la MCC, el bosque de encino es el que domina y presenta la mayor riqueza florística. En trabajos previos se han reportado hasta cuatro diferentes tipos de encinares para la sierra de Álvarez (García-Sánchez et al. 1999, ZavalaChávez \& García-Sánchez 1999), y es posible que su interacción con el resto de los tipos de vegetación incida en $\mathrm{su}$ alta riqueza. Estos autores han resaltado la presencia de encinares mesófilos dentro del área, caracterizada por la presencia de Asclepias pellucida, Quercus affinis, Q. crassipes y Tilia americana var. mexicana (GarcíaSánchez et al. 1999), que coinciden con algunas zonas húmedas de bosque de encino dentro de la MCC pero difieren de lo que aquí denominamos el bosque afín a mesófilo de montaña.

El bosque de galería es el tipo de vegetación con mayor diversidad taxonómica a nivel de familia, el segundo en riqueza florística y presenta diferencias en su composición, según el sustrato geológico y la altitud. A pesar de que Platanus mexicana es constante en el bosque de galería, las áreas con roca ígnea que colindan con el bosque de encinopino, presentan también Alnus acuminata subsp. arguta, así como las áreas colindantes con el matorral submontano presentan Carya illinoinensis. Se ha mencionado que en las zonas rivereñas existen ecotonos de alta diversidad biológica y de funcionamiento complejo por su posición entre los sistemas acuático y terrestre (Mendoza et al. 2014) como se observa dentro de la MCC y áreas adyacentes en cuanto su riqueza de especies y diversidad de asociaciones vegetales.

El bosque de encino-pino, que ocupa el tercer lugar en riqueza y diversidad taxonómica, se encuentra consignado a la región sur-sureste de la MCC, en las zonas más altas del área, de 1,580 a 2,300 m snm y con sustrato derivado de rocas ígneas. En este tipo de vegetación existen manchones reducidos de pequeñas herbáceas que parecen indicar un microambiente de alta humedad, entre estos destacan los elementos herbáceos Cranichis subumbellata, Cyclopogon violaceus y Cyclopogon luteo-albus, reconocida esta última como indicadora de calidad ambiental en otros tipos de vegetación como el bosque mesófilo de montaña (DíazToribio 2009). En regiones cercanas, como el Parque Nacional El Potosí, la riqueza de especies del bosque de pino-encino se sitúa por encima de la que presenta el bosque de encino (Enríquez-Salaices-Valdez 2019).

Tabla 4. Proporción de formas de crecimiento en la MCC por tipo de vegetación y total.

\begin{tabular}{|c|c|c|c|c|c|c|}
\hline $\begin{array}{l}\text { Forma de } \\
\text { crecimiento }\end{array}$ & $\begin{array}{l}\text { Bosque de } \\
\text { encino }(\%)\end{array}$ & $\begin{array}{c}\text { Bosque de } \\
\text { encino-pino }(\%)\end{array}$ & $\begin{array}{c}\text { Bosque de } \\
\text { galería }(\%)\end{array}$ & $\begin{array}{c}\text { Bosque afín a } \\
\text { mesófilo de montaña } \\
(\%)\end{array}$ & $\begin{array}{c}\text { Matorral } \\
\text { submontano (\%) }\end{array}$ & TOTAL (\%) \\
\hline$\overline{\text { Árbol }}$ & 13.60 & 14.21 & 16.74 & 35.29 & 13.95 & 14.67 \\
\hline Arbusto & 17.20 & 16.59 & 14.88 & 11.76 & 27.32 & 18.90 \\
\hline Hierba & 57.60 & 62.55 & 57.67 & 33.30 & 44.18 & 57.61 \\
\hline Epífita & 3.60 & 3.31 & 4.65 & 9.80 & 5.23 & 3.12 \\
\hline Trepadora & 8.00 & 3.31 & 6.04 & 9.80 & 9.30 & 5.68 \\
\hline
\end{tabular}


Flora de la microcuenca del Cañón de Los Chivos

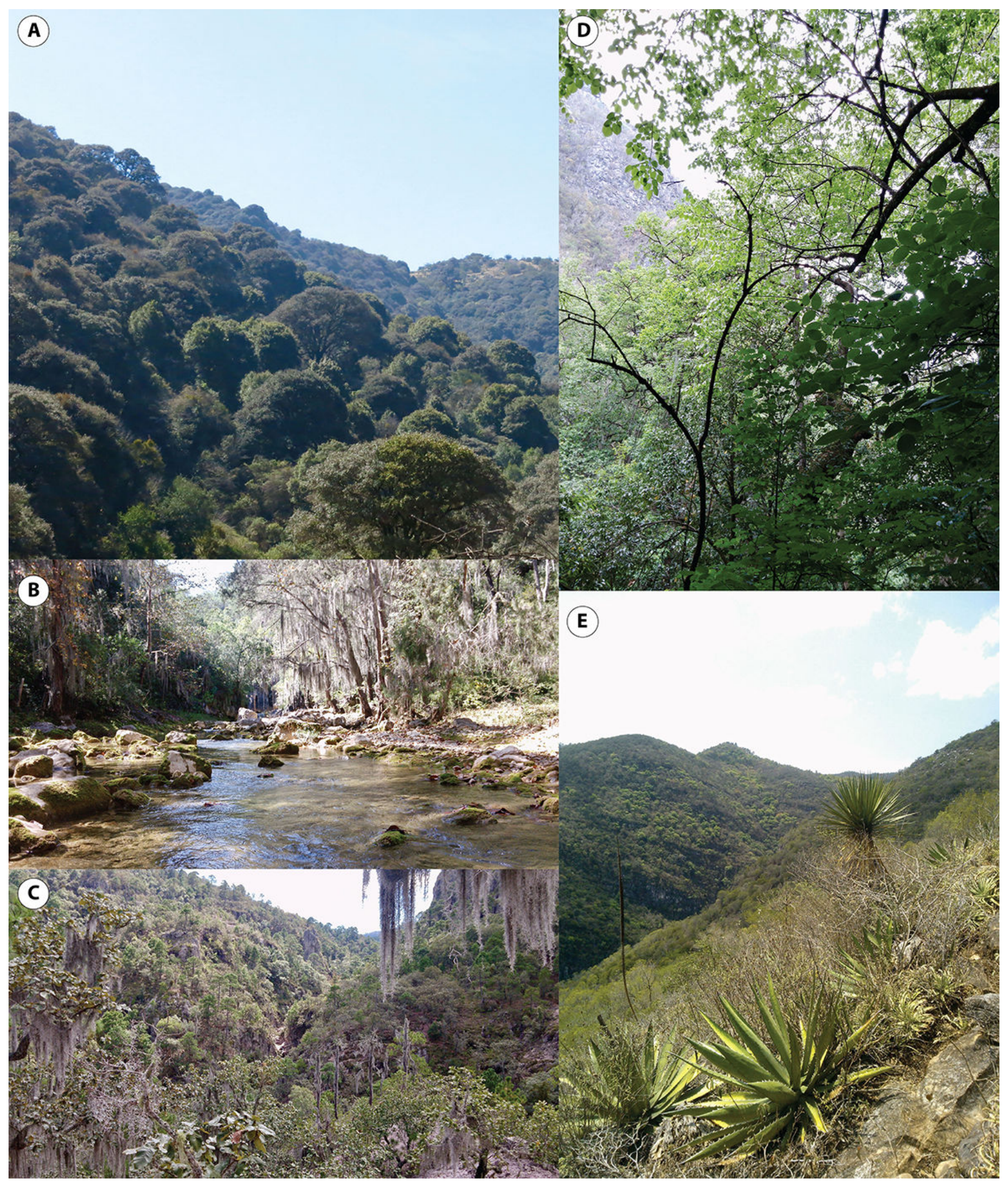

Figura 6. Tipos de vegetación en la MCC, San Luis Potosí, México. A) Bosque de encino; B) Bosque de galería; C) Bosque de encino-pino; D) Bosque afín a mesófilo de montaña; E) Matorral submontano. Fotos J.I. Morales de la T. 
El matorral submontano es de los tipos de vegetación de menor riqueza florística en la MCC, en contraste con lo reportado para otras regiones de San Luis Potosí, como la sierra de Guadalcázar, en dónde es el tipo de vegetación más rico en especies (Torres-Colín et al. 2017). La menor riqueza del matorral submontano, respecto a la mayoría de los bosques templados de la MCC, probablemente se asocia a la menor área que éste ocupa en la microcuenca, lo cual reduce los escenarios ambientales que favorecerían su riqueza. Respecto a las variantes de composición observadas en este tipo de vegetación fue notorio Esenbeckia berlandieri en las zonas más húmedas y protegidas de la alta incidencia solar, donde destaca como arbolito vigoroso, e incluso llega a formar pequeños rodales, en contraste con las áreas escarpadas y más xéricas con especies de menor altura, donde Painteria elachistophylla fue la especie más frecuente. Esto coincide con lo reportado para el matorral submontano en Linares, Nuevo León, donde algunas especies, generalmente de porte arbustivo, pueden llegar a crecer como árboles o arbolitos en áreas protegidas de la alta radiación solar con microclimas más húmedos (García-Hernández \& Jurado 2008). Así mismo, Myrtillocactus geometrizans y Stenocereus queretaroensis son notorios en las zonas con suelos derivados de roca ígnea, dentro del matorral submontano, mientras que, en las áreas con substrato sedimentario, está ausentes, tal y como fue descrito por

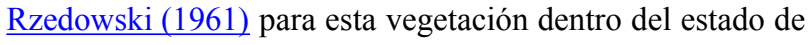
San Luis Potosí.

Tabla 5. Flora vascular representada por tipo de vegetación en la MCC, San Luis Potosí, México.

\begin{tabular}{|c|c|c|c|c|}
\hline Tipo de vegetación & Familias & Géneros & Especies & $\%$ \\
\hline Bosque de encino & 75 & 182 & 250 & 45.87 \\
\hline Bosque de galería & 73 & 170 & 215 & 39.45 \\
\hline Bosque de encino-pino & 62 & 149 & 211 & 38.72 \\
\hline Matorral submontano & 60 & 143 & 172 & 31.75 \\
\hline $\begin{array}{l}\text { Bosque afín a mesófilo de } \\
\text { montaña }\end{array}$ & 34 & 48 & 51 & 9.35 \\
\hline
\end{tabular}

La menor riqueza florística presente en el tipo de vegetación afín a bosque mesófilo de montaña está asociada a su extensión, ya que se restringe a un pequeño manchón de 2.1 ha protegido dentro de una cañada en una ladera de exposición noroeste. Este tipo de vegetación fue designado como bosque afín a mesófilo de montaña ya que los elementos vegetales presentes en el sitio son característicos de este tipo de vegetación, poco comunes en los bosques de encino de la MCC, por lo que este pequeño rodal se distingue de la matriz que lo rodea. En este tipo de vegetación se percibe una alta humedad, diferente a la de su entorno, debido al arroyo semipermanente que lo cruza y por su ubicación dentro de una pequeña cañada oscurecida, que le confiere un microambiente con una constante presencia de neblina y otros tipos de condensación. Sus principales componentes arbóreos son Persea americana, Tilia americana var. mexicana, Cinnamomum pachypodum, Carya ovata y Morus celtidifolia que han sido asociados previamente a bosques mesófilos de montaña en México (Rzedowski 1996, Villaseñor 2010) y particularmente en la SMOr, como los de Copalillos y Rioverde en el estado de San Luis Potosí, Landa de Matamoros en el estado de Querétaro y la Reserva de la Biosfera El Cielo en el estado de Tamaulipas (Puig et al. 1987, Cartujano et al. 2002, Salinas-Rodríguez \& Cruzado-Cortés 2011, FortanelliMartínez et al. 2014). Previamente se ha indicado que la presencia de bosques húmedos, como el bosque afín a mesófilo de montaña, en la sierra de Álvarez, es favorecida por la neblina y otros tipos de condensación de humedad derivados del efecto de barlovento provocado por el viento que proviene del Golfo de México (Cserna \& Bello 1963, Villaseñor 2010, Salinas-Rodríguez \& Cruzado-Cortés 2011). Su registro en la MCC representa su distribución más continental dentro del estado de San Luis Potosí hasta el momento y abre la posibilidad de encontrar otros fragmentos en la región que representen refugios para su conservación.

Formas de crecimiento. La proporción de la riqueza de especies, con respecto a las formas de crecimiento en la MCC, coincide con lo reportado para otras regiones con ecosistemas de montaña en la SMOr, como el Parque Nacional El Potosí y la sierra de Zapalinamé, en donde dominan las herbáceas, seguidas por los arbustos, árboles y en menor proporción las trepadoras y epífitas (EncinaDomínguez et al. 2009, Enríquez-Salaices-Valdez 2019). A pesar de que, a nivel de diversidad gamma, en las formas de crecimiento, se tuvo un mayor porcentaje de arbustos respecto a los árboles, en el bosque de galería y el fragmento afín a bosque mesófilo de montaña se presentó un mayor porcentaje de árboles con relación a los arbustos. Este efecto puede atribuirse al grado de conservación en estos tipos de vegetación, sobre todo en el afín a bosque mesófilo de montaña, donde además se presentó el mayor porcentaje de trepadoras y epifitas. Estas últimas son un indicador de humedad y conservación en algunos tipos de vegetación debido a las preferencias de estas formas de vida (Lüttge 1997, Köster et al. 2009).

Endemismos, especies en riesgo y nuevos registros. De la riqueza florística presente en México, casi la mitad son endémicas de sus provincias biogeográficas (Villaseñor $\underline{2003}$, 2004). El endemismo en la MCC equivale al 1.55\% de las 11,600 especies endémicas de México (Villaseñor 2016) y al $15.94 \%$ de las 1,135 endémicas de la SMOr 
(Salinas-Rodríguez et al. 2017). Las familias más representativas son Asteraceae y Asparagaceae con cinco especies, Crassulaceae con cuatro especies y Cactaceae con tres especies. Asteraceae y Cactaceae son también las familias más ricas en especies endémicas dentro de la SMOr (Salinas-Rodríguez et al. 2017). Cabe destacar que Ceratozamia zaragozae, Tigridia potosina y Verbesina sororia, tienen una distribución restringida dentro del estado de San Luis Potosí (Villaseñor 2016, Castillo-Lara et al. 2017). De las 117 especies incluidas en alguna de las listas de protección, únicamente 11 especies están registradas en alguna categoría de riesgo dentro de la NOM-059 (SEMARNAT 2010). Algunas como C. zaragozae y Schiedeella nagelii son endémicas a San Luis Potosí y regiones adyacentes (Villaseñor 2016, Castillo-Lara et al. 2017, De-Nova et al. 2018) y C. zaragozae se encuentra en la categoría de peligro de extinción en la NOM-059(SEMARNAT 2010), en el apéndice 1 de la CITES y en la categoría de peligro crítico (CR) de la IUCN. El bajo número de especies catalogadas en las categorías de riesgo de la NOM-059 con respecto a la CITES y IUCN, resalta la necesidad de actualizar la norma nacional y realizar un análisis ecológico más minucioso (Enríquez-SalaicesValdez 2019). Los seis nuevos registros para el estado de San Luis Potosí han sido de relevancia puesto que existen pocos registros para México. Cinco de los nuevos registros pertenecen a la familia Orchidaceae y uno a la familia Iridiaceae. Además, se comprobó la presencia de Staphylea pringlei, la cual había sido previamente sugerida para el estado (Carranza-González 2004) y aunque recientemente ha sido mencionada para Rioverde, San Luis Potosí (Salinas-Rodríguez et al. 2017), la presente investigación es la primera que incluye recolectas formales respaldadas con ejemplares de herbario en una colección. La población de S. pringlei en la MCC se encuentra confinada dentro del manchón afín a bosque mesófilo de montaña, con algunos individuos dispersos a lo largo de un arroyo adyacente en un segmento no mayor a $1 \mathrm{~km}$. Su presencia es bastante significativa, ya que se ha considerado una especie relictual, con afinidad por los bosques mesófilos de montaña y con algunas pocas poblaciones en el Cañón de Iturbide y el Parque Nacional Cumbres de Monterrey, Nuevo León, en el bosque mesófilo de montaña de Tamaulipas, en los límites entre Hidalgo y Veracruz, y en algunos bosques mesófilos de montaña de Veracruz. (Marroquín 1976, Sosa 1988, Avendaño \& Durán 2000, Cavazos 2000, Salinas-Rodríguez et al. 2013). Lo anterior indica que la MCC podría representar un refugio para otras especies de distribución restringida.

La MCC es un eslabón importante para la conservación de la biodiversidad dentro del estado de San Luis Potosí y para El Corredor Ecológico SMOr, debido a que representa un área de importante diversidad florística y permite la conexión entre áreas naturales protegidas como son el Parque Nacional El Potosí y el Área Natural de Protección de Flora y Fauna Sierra de Álvarez. Su heterogeneidad ambiental, promovida por su complejidad de geoformas y ubicación geográfica, resalta a la MCC como refugio de biodiversidad, con presencia de endemismos regionales, elementos de ecosistemas relictuales, así como comunidades vegetales en buen estado de conservación. Todo esto soporta que la MCC sea propuesta como área destinada a la conservación.

\section{Agradecimientos}

Agradecemos al Consejo Nacional de Ciencia y Tecnología por el apoyo económico e infraestructura que permitió realizar esta investigación con el proyecto CONACYT CB-2014/243454 otorgados a JADNV. El primer autor agradece a la UASLP por el apoyo como becario 30784 dentro del Herbario SLPM y al IIZD-UASLP por todas las facilidades proporcionadas durante el desarrollo del proyecto, además del apoyo durante el trabajo de campo de Cesar Camarillo H, Estefanía G. Fernández P., Alejandra Berenice Ibarra H., Brianda Fernanda Martínez B., Paulina Rodríguez M., Israel Rodríguez O., Eunice Salazar A., Guillermo Javier Sánchez F. Se agradece a José García Pérez, Eleazar Carranza González, Javier Fortanelli Martínez y Juan Antonio Reyes Agüero por la identificación de algunos especímenes botánicos.

\section{Literatura citada}

Andrade M, Calderón de Rzedowski G, Camargo-Ricalde SL, Grether R, Hernández HM, Martínez-Bernal A, Rico L, Rzedowski RJ, Sousa M. 2007. Familia Leguminosae, subfamilia Mimosoideae. Flora del Bajio y de Regiones Adyacentes 150: 1-230.

APG [Angiosperm Phylogeny Group]. 2016. An update of the Angiosperm Phylogeny Group classification for the orders and families of flowering plants: APG IV. Botanical Journal of the Linnean Society 181: 1-20. DOI: https://doi.org/10.1111/boj.12385

Avendaño S, Durán C. 2000. Se confirma la presencia de Staphylea pringlei S. Watson (Staphyleaceae) en Veracruz. La Ciencia y el Hombre 1: 83-84.

Ávila-Sánchez P, Sánchez-González A, Catalán EC. 2010. Estructura y composición de la vegetación del Cañón del Zopilote, Guerrero, México. Revista Chapingo. Series, Ciencias Forestales y del Ambiente 2: 119-138. DOI: http://dx.doi.org/10.5154/r.rchscfa.2010.02.003

Ballard HE. 1994. Familia Violaceae. Flora del Bajío y de Regiones Adyacentes 31: 1-38. 
Borhidi A. 2006. Rubiáceas de México. Budapest: Akadémiai Kiadó. ISBN: 9630582651

Calderón de Rzedowski G, Rzedowski J. 1994. Familia Smilacaceae. Flora del Bajio y de Regiones Adyacentes 26: $1-23$.

Calderón de Rzedowski G, Rzedowski RJ. 2005. Flora fanerogámica del Valle de México. Pátzcuaro, Michoacán, México: Instituto de Ecología AC. Comisión Nacional para el Conocimiento y Uso de La biodiversidad. ISBN: 9709000179

Calderón de Rzedowski G, Rzedowski RJ. 2006. Familia Sapindaceae. Flora del Bajío y de Regiones Adyacentes. 142: 1-70.

Carranza-González E, Madrigal-Sánchez X. 1995. Familia Betulaceae. Flora del Bajio y de Regiones Adyacentes 39: 1-32.

Carranza-González E. 2004. Familia Staphyleaceae. Flora del Bajio y de Regiones Adyacentes 122: 1-11.

Carranza-González E. 2007. Familia Convolvulaceae I. Flora del Bajío y de Regiones Adyacentes 151: 1-131.

Carranza-González E . 2008. Familia Convolvulaceae II. Flora del Bajío y de Regiones Adyacentes 155: 1-109.

Carrasco B. 1970. La Formación El Abra (Formación El doctor) en la Plataforma Valles-San Luis Potosí. Revista del Instituto Mexicano de Petróleo 2: 97-99.

Carrillo-Bravo J. 1971. La Plataforma Valles-San Luis Potosí. Boletín de la Asociación Mexicana de Geólogos Petroleros 23: 1-106.

Cartujano S, Zamudio S, Alcántara O, Luna I. 2002. El bosque mesófilo de montaña en el municipio de Landa de Matamoros, Querétaro, México. Boletín de la Sociedad Botánica de México 70: 13-43. DOI: https:// doi.org/10.17129/botsci.1653

Carvajal S. 2007. Familia Moraceae. Flora del Bajío y de Regiones Adyacentes 147: 1-59.

Castillo-Gómez HA. 2015. Flora vascular, vegetación y plantas útiles del Cañón del Espinazo del Diablo. San Luis Potosí, México. MSc. Thesis. Universidad Autónoma de San Luis Potosí.

Castillo-Lara P. 2003. Encinares de la Sierra de Álvarez: caracterización y dinámica. MSc. Thesis. Universidad Autónoma Agraria Antonio Narro.

Castillo-Lara P. 2007. Evolución reciente y estado actual de la vegetación del área natural protegida Sierra de Álvarez, S.L.P. PhD. Thesis. Universidad Autónoma de san Luis Potosí.

Castillo-Lara P, Aguilar PO, De-Nova JA. 2017. Ceratozamia zaragozae Medellín-Leal (Zamiaceae), an endangered Mexican Cycad: New information on population structure and spatial distribution. Britonia 70: 155-165. DOI: https://doi.org/10.1007/s12228-017-9513 $\underline{-1}$
Cavazos CC. 2000. Evaluación del bosque mesófilo de montaña de San Carlos, Tamaulipas. MsC. Thesis. Universidad Autónoma de Nuevo León.

Cayuela L, Granzow-de la Cerda I, Albuquerque FS, Golicher DJ. 2012. Taxonstand: Taxonomic Standardization of Plant Species Names. Methods in Ecology and Evolution 6: 1078-1083. DOI: https://doi. org/10.1111/j.2041-210X.2012.00232.x

Cervantes-Zamora Y, Cornejo Olguín SL, Lucero-Márquez R, Espinoza-Rodríguez JM, Miranda Víquez E, PinedaVelázquez A. 1990. Provincias Fisiográficas de México, Atlas Nacional de México. México: Instituto de Geografía, Universidad Nacional Autónoma de México. http://conabio.gob.mx/informacion/metadata/gis/rfisio4m gw.xml? xsl=/db/metadata/xsl/fgdc html.xsl\&_indent $=$ n o (Accessed December 3, 2018)

Christenhusz MJM, Zhang X, Schneider H. 2011. A linear sequence of extant families and genera of lycophytes and ferns. Phytotaxa 19: 7-54. DOI: http://dx.doi.org/10.1164 6/phytotaxa.19.1.2

CITES. 2019. The Convention on International Trade in Endangered Species of Wild Fauna and Flora. Checklist of CITES species. http://checklist.cites.org/ (Accessed March 15, 2019).

Colwell RK, Chao A, Gotelli NJ, Lin SY, Mao CX, Chazdon RL, Longino JT. 2012. Models and estimators linking individual-based and sample-based rarefaction, extrapolation, and comparison of assemblages. Journal of Plant Ecology 5: 3-21. DOI: https://doi.org/10.109 3/jpe/rtr044

CONABIO [Comisión Nacional para el Conocimiento y Uso de la Biodiversidad]. 2019. Portal Naturalista. http:// www.naturalista.mx (Accessed August 13, 2019).

Croat TB, Carlsen M. 2003. Familia Araceae. Flora del Bajío y de Regiones Adyacentes 114: 1-41.

Cserna GE, Bello BA. 1963. Geología de la parte central de la Sierra de Álvarez, municipio Zaragoza, Estado de San Luis Potosí. Boletín del Instituto de Geología (Universidad Nacional Autónoma de México) 2: 23-63.

Daniel TF, Acosta-Castellanos S. 2003. Familia Acanthaceae. Flora del Bajio y de Regiones Adyacentes 117: 1-177.

De-Nova JA. 2018. La diversidad florística potosina, un patrimonio que debemos conservar. Universitarios Potosinos 223: 4-10.

De-Nova JA, Castillo-Lara P, Gudiño-Cano AK, García PJ. 2018. Flora endémica del estado de San Luis Potosí y regiones adyacentes en México. AridoCiencia 3: 21-41.

Díaz-Toribio MH. 2009. Orquídeas terrestres como indicadoras de calidad ambiental en fragmentos de bosque mesófilo de montaña. MSc. Thesis. Instituto de Ecología, AC. 
Egan P, Price M. 2014. Las montañas como torres de agua del mundo: Protegiendo el agua y los servicios ecosistémicos de montaña ante el cambio climático. Informe de política. UICN Comisión para la Gestión de Ecosistemas. https://portals.iucn.org/library/sites/library/ files/documents/2014-050-Es.pdf (Accessed September 1, 2018).

Encina-Domínguez JA, Zárate-Lupercio AE, Castillón E, Valdés-Reyna J, Villarreal-Quintanilla JA. 2009. Composición y aspectos estructurales de los bosques de encino de la Sierra de Zapalinamé, Coahuila, México. Acta Botanica Mexicana 86: 71-108. DOI: https:// doi.org10.21829/abm86.2009.1078

Enríquez-Salaices-Valdez E. 2016. Diversidad filogenética de los encinares del Área Natural de Protección de Flora y Fauna Sierra de Álvarez. BSc. Thesis. Universidad Autónoma de San Luis Potosí.

Enríquez-Salaices-Valdez E. 2019. Inventarios para la conservación: Flora vascular del Área Natural Protegida Parque Nacional El Potosí. MSc. Thesis. Universidad Autónoma de San Luis Potosí.

Espejo-Serna A, García-Cruz J, López-Ferrari AR, Jiménez MR, Sánchez SL. 2002. Orquídeas del Estado de Morelos. Orquidea 1: 1-392.

Espejo-Serna A, López-Ferrari AR. 2003. Familia Alliaceae. Flora de Veracruz 132: 1-22.

Espejo-Serna A, López-Ferrari AR, Ceja-Romero J. 2009. Familia Commelinaceae. Flora del Bajio y de Regiones Adyacentes. 162: 1-122.

Espejo-Serna A, López-Ferrari AR., Ceja-Romero J. 2010a. Familia Iridaceae. Flora del Bajio y de Regiones Adyacentes 166: 1-81.

Espejo-Serna A, López-Ferrari AR, Ramírez-Morillo I. 2010b. Familia Bromeliaceae. Flora del Bajio y Regiones Adyacentes 165: 1-82.

Espinosa-Jiménez JA, López-Cruz A, Pérez-Farrera MA, López S. 2014. Inventario florístico de la cañada La Chacona-Juan Crispín y zonas adyacentes, depresión Central de Chiapas, México. Botanical Sciences 92: 205-241. DOI: https://doi.org/10.17129/botsci.30

Farjon A. 1996. Biodiversity of Pinus (Pinaceae) in Mexico: speciation and palaeo-endemism. Botanical Journal of the Linnean Society 121: 365-384. DOI: https://doi.org/10.1111/j.1095-8339.1996.tb00762.x

Fernández-Nava R. 1996. Familia Rhamnaceae. Flora del Bajio y de Regiones Adyacentes 43: 1-68.

Flores MG, Jiménez LJ, Madrigal SX, Moncayo RF, Takaki TF. 1971. Memoria del mapa de tipos de vegetación de la República Mexicana. México, DF: Secretaría de Recursos Hidráulicos https://es.scribd.com/document/ 361025952/Memoria-Del-Mapa-de-Tipos-de-Vegetacion -de-La-Republica-Mexicana (Accessed June 16, 2019)
Font Quer P. 1953. Diccionario de Botánica. Barcelona, España: Peninsula. ISBN: 8433558048

Fortanelli-Martínez J, García-Pérez J, Castillo-Lara P, 2014. Estructura y composición de la vegetación del bosque de niebla de Copalillos, San Luis Potosí, México. Acta Botanica Mexicana 106: 161-186. DOI: https://doi.org/ $\underline{10.21829 / \mathrm{abm} 106.2014 .218}$

Fryxell PA. 1993. Familia Malvaceae. Flora del Bajío y de Regiones Adyacentes 16: 1-74.

García E. 1981. Modificaciones al sistema de clasificación climática de Köppen. Ciudad de México:Instituto de Geografía, Universidad Nacional Autónoma de México.

García-Hernández J, Jurado E. 2008. Caracterización del matorral con condiciones prístinas en Linares N.L., México. Ra Ximhai 1: 1-21.

García LE, Koch SD. 1995. Familia Compositae. Tribu Cardueae. Flora del Bají y de Regiones Adyacentes 32: $1-53$.

García-Mendoza AJ. 2004. Agaváceas. In: García-Mendoza AJ, Ordoñez MJ, Briones-Salas M, eds. Biodiversidad de Oaxaca. México, DF: Instituto de Biología-Universidad Nacional Autónoma de México - Fondo Oaxaqueño para la Conservación de la Naturaleza-World Wildlife Fund, pp. 159-169. ISBN: 9703220452

García-Sánchez F, Aguirre RJ, Villanueva DJ, García-Pérez J. 1999. Contribución al conocimiento florístico de la Sierra de Álvarez, San Luis Potosí, México. Polibotánica 10: 73-103.

García-Sánchez F, Aguirre RJ. 2011. Guía de campo para la identificación de los árboles de Sierra de Álvarez, S.L.P. San Luis Potosí, México: Universidad Autónoma de San Luis Potosí.

Gentry HS. 1982. Agaves of continental North America. Tucson: University of Arizona Press. ISBN: 0816523959; 978-0816523955

González CO, Giménez de AJ, García-Pérez J, Aguirre R. 2007. Flórula vascular de la Sierra de Catorce y territorios adyacentes, San Luis Potosí, México. Acta Botanica Mexicana 78: 1-38. DOI: https://doi.org/10. 21829/abm78.2007.1027

González-Elizondo MS, González-Elizondo M. 2015. Familia Ericaceae. Flora del Bajio y Regiones Adyacentes 183: 1-128.

González-Oreja JA, De la Fuente-Díaz-Ordaz AA, Hernández-Santín L, Buzo-Franco D, Bonache-Regidor C. 2010. Evaluación de estimadores no paramétricos de la riqueza de especies. Un ejemplo con aves en áreas verdes de la ciudad de Puebla, México. Animal Biodiversity and Conservation 1: 31-45.

Govaerts R, Frodin DG. 1998. World Checklist and Bibliography of Fagales. Richmond, U.K.: Royal Botanic Gardens, Kew. ISBN: 9781900347464 
Hernández-Sandoval L. 2019. Dos especies nuevas de Nolina (Asparagaceae) del centro de México. Phytoneuron 12: 1-9.

Herrera Y. 2001. Las Gramineas de Durango. México, DF: Instituto Politécnico Nacional y Comisión Nacional para el Conocimiento y Uso de la Biodiversidad. ISBN: 0187-7151.

Hoch PC, Berry PE, Wagner WL, Zardini E. 2009. Familia Onagraceae. Flora Mesoamericana 1: 345-359. ISBN: 968-36-3309-9

Ibarra-Manríquez G, Cornejo-Tenorio G, GonzálezCastañeda N, Piedra-Malagón EM, Luna A. 2012. El género Ficus L. (Moraceae) en México. Botanical Sciences 90: 389-452. DOI: https://doi.org/10.17129/ botsci.472

INEGI [Instituto Nacional de Estadística y Geografía]. 1987. Carta de climas. Hoja México. (escala 1:1000,000). México, DF. https://www.inegi.org.mx/ temas/climatologia// (Accessed September 1, 2019).

INEGI. 2001. Síntesis geográfica del estado de San Luis Potosí. Aguascalientes México. ISBN: 970-13-3736-X

INEGI. 2013. Datos para Sistemas de Información Geográfica de sistemas hidrológicos, Simulador de Flujos de agua en cuencas hidrográficas (SIATL). http:// antares.inegi. org.mx/analisis/red_hidro/siatl// (Accessed September 1, 2018).

IUCN [The International Union for Conservation of Nature]. 2019. The International Union for Conservation of Nature. Red list of threatened species. https://ww w.iucn.org/ (Accessed March 15, 2019).

Körner C, Spehn E. 2002. Mountain biodiversity: a global assessment. London: The Parthenon Publishing Group. ISBN: 9781842140918

Köster N, Friedrich K, Nieder J, Barthlott W. 2009. Conservation of epiphyte diversity in an Andean landscape transformed by human land use. Conservation Biology 23: 911-919. DOI: https://doi.org/10.1111/j.15 23-1739.2008.01164.x

López-Doncel R. 2002. Estudios Estratigráficos y Deposicionales de la Formación Soyatal (Turoniano), en la Sierra de Álvarez y Sierra del Coro, porción centrooccidental del Estado de San Luis Potosí: Geos, Boletín Informativo de la Unión Geofisica Mexicana 1: 335-336.

Lorea-Hernández FG. 2004. Familia Capparaceae. Flora del Bajio y de Regiones Adyacentes 130: 1-37.

Lot A, Chiang F. 1986. Manual de herbario. Administración y manejo de colecciones, técnicas de recolección y preparación de ejemplares botánicos. México: Consejo Nacional de la Flora de México. ISBN: 9686144005; 9789686144000

Luna I, Morrone J, Espinosa D, eds. 2004. Biodiversidad de la Sierra Madre Oriental. México DF: Universidad
Nacional Autónoma de México, Facultad de Ciencias. ISBN: 970-32-1526-2

Lüttge U. 1997. Physiological ecology of tropical plants. Germany: Springer-Verlag Berlin Heidelberg. ISBN: 978-3-540-71793-5

Marroquín JS. 1976. Vegetación y florística del nordeste de México. I. Aspectos sinecológicos en Coahuila. Revista de la Sociedad Mexicana de Historia Natural 36: 69-101.

Martínez G. 2003. Utilización de la fauna silvestre en la Sierra de Álvarez, San Luis Potosí. MSc. Thesis. Instituto de ecología, AC.

Martínez-Gordillo M, Fragoso-Martínez I, García-Peña M, Montiel O. 2013. Géneros de Lamiaceae de México, diversidad y endemismo. Revista Mexicana de Biodiversidad 84: 30-86. DOI: https://doi.org/10.7550/ rmb.30158

Martínez M. 2015. Ranunculaceae. Flora del Bajío y Regiones Adyacentes 190: 1-76.

Mendoza CM, Quevedo NA, Bravo VÁ, Flores MH, De la Isla de Bauer M, Gavi RF, Zamora MB. 2014. Estado ecológico de ríos y vegetación ribereña en el contexto de la nueva Ley General de Aguas de México. Revista Internacional de Contaminación Ambiental 4: 429-436.

Mendoza-Ruiz A, Pérez-García B. 2009. Helechos y licopodios de México. Vol. 1. México, DF: Comisión Nacional para el Conocimiento y Uso de la Biodiversidad y Universidad Autónoma Metropolitana, Iztapalapa. ISBN: 978-607-7607-01-4

Mickel JT, Smith A. 2004. The Pteridophytes of Mexico. Memoirs of the New York Botanical Garden 1: 1-1054.

Palacios GR. 2008. El Piojito, Ferrocarril El Potosí y Rioverde 1898-1949. SLP, México:. Cocodrilo Atrabiliario. https://xdoc.mx/documents/el-piojito-ferro carril-el-potosi-y-rioverde-1898-1949-5c12bc9d7495e

(Accessed September 1, 2018).

Pérez-Calix E, Carranza-González E . 2005. Familia Hydrophyllaceae. Flora del Bajio y de Regiones Adyacentes 139: 1-56.

Pinzón JP, Ramírez-Morillo IM, Carnevali G, Barfuss MH, Till W, Tun J, Ortiz-Díaz JJ. 2016. Phylogenetics and evolution of the Tillandsia utriculata complex (Bromeliaceae, Tillandsioideae) inferred from three plastid DNA markers and the ETS of the nuclear ribosomal DNA. Botanical Journal of the Linnean Society 181: 362-390. DOI: https://doi.org/10.1111/boj. $\underline{12425}$

Pool A, Knapp S. 2012. Familia Lamiaceae. In: Davidse, G., M. Sousa S., S. Knapp y F. Chiang, eds. Flora Mesoamericana Vol. 4, núm. 2. St. Louis, USA. Missouri Botanical Garden Press. ISBN: 1935641085

Puig H, Bracho R, Sosa VJ. 1987. El bosque mesófilo de montaña: composición florística y estructura. In: Puig, 
Bracho HR, eds. El Bosque Mesófilo de Montaña de Tamaulipas. México DF: Instituto de Ecología AC, pp. 55-79. ISBN: 9687213078; 9789687213071

R Development Core Team. 2019. R: A language and environment for statistical computing. Vienna: $R$ Foundation for Statistical Computing. https://www.Rproject.org/ (Accessed February 6, 2019).

Reyes-Agüero JA, González-Medrano F, García-Pérez JD. 1996. Flora vascular de la Sierra Monte Grande, municipio de Charcas, San Luis. Potosí, México. Boletín de la Sociedad Botánica de México 58: 31-42. DOI: https://doi.org/10.17129/botsci.1485

Rzedowski J. 1961. Vegetación del Estado de San Luis Potosi. PhD. Thesis. Universidad Nacional Autónoma de México.

Rzedowski J. 1972. Contribuciones a la fitogeografía florística e histórica de México III. Algunas tendencias en la distribución geográfica y ecológica de las Compositae mexicanas. Ciencia 27: 123-132.

Rzedowski J. 1978. Vegetación de México. México DF: Limusa. ISBN: 968-18-0002-8

Rzedowski J. 1996. Análisis preliminar de la flora vascular de los bosques mesófilos de montaña de México. Acta Botanica Mexicana 35: 25-44. DOI: https://doi.org/10.2 $\underline{1829 / \mathrm{abm} 35.1996 .955}$

Rzedowski J, Calderón de Rzedowski G. 1997. Familia Leguminosae Subfamilia Caesalpinioideae. Flora del Bajio y Regiones Adyacentes 51: 1-111.

Rzedowski J, Calderón de Rzedowski G. 2002. Familia Verbenaceae. Flora del Bajío y de Regiones Adyacentes 100: 1-145.

Rzedowski J, Calderón de Rzedowski G. 2005. Familia Vitaceae. Flora del Bajio y de Regiones Adyacentes 131: 1-37.

Rzedowski J. Calderón de de Rzedowski G . 2011. Familia Viscaceae. Flora del Bajío y de Regiones Adyacentes 170: $1-59$.

Rzedowski J, Calderón de Rzedowski G, Carrillo RP. 2011. Familia Compositae, Tribu Heliantheae II (géneros Lagascea-Zinnia). Flora del Bajío y de Regiones Adyacentes 172: 1-420.

Rzedowski J. 2015. Catálogo preliminar de las especies de árboles silvestres de la Sierra Madre Oriental. Flora del Bajio y de Regiones Adyacentes. Fascículo Complementario 30: 1-378.

Rzedowski J, Calderón de Rzedowski G, Torres CL, Grether R. 2016. Familia Leguminosae, Subfamilia Papilionoideae (Aeschynomene-Diphysa). Flora del Bajio y Regiones Adyacentes 192: 1-330.

Salazar GA. 1990. Catasetum integerrimum. In: Hágsater E, Salazar GA, eds. Icones Orchidacearum, Orchids of Mexico part 1. México, DF: Asociación Mexicana de Orquideologia, AC. ISBN: 0188-4018
Salinas-Rodríguez M, Estrada-Castillón E, VillarrealQuintanilla JA. 2013. Flora and phytogeography of the canon de Iturbide, Nuevo Leon, Mexico. Journal of the Botanical Research Institute of Texas 7: 803-819.

Salinas-Rodríguez M, Cruzado-Cortés J. 2011. Nota sobre un fragmento de bosque mesófilo de montaña del municipio de Río Verde, San Luis Potosí. Boletín de la Sociedad Botánica de México 89: 126-128. DOI: https:// doi.org/10.17129/botsci.374

Salinas-Rodríguez M. 2015. Conocimiento, manejo y conservación de la diversidad florística de la Sierra Madre Oriental, México. PhD. Thesis. Universidad Autónoma de León.

Salinas-Rodríguez M, Estrada-Castillón E, VillarealQuintanilla JA. 2017. Endemic vascular plants of the Sierra Madre Oriental, Mexico. Phytotaxa 1: 1-52. DOI:

Sánchez H, Soberanes F. 2001. Carta geológico-minera La Salitrera, F14-C15, San Luis Potosí. Pachuca, Hidalgo: Servicio Geológico Mexicano. Secretaria de Economía. http://www.sgm.gob.mx/cartas/Cartas Ed50.jsp

(Accessed September 1, 2018).

Sánchez-Ken JG. 2019. Riqueza de especies, clasificación y listado de las gramíneas (Poaceae) de México. Acta Botanica Mexicana 126: 1-73. DOI: https://doi.org/10.21 $\underline{829 / \mathrm{abm} 126.2019 .1379}$

SEMARNAT [Secretaría del Medio Ambiente y Recursos Naturales], 2010. Norma Oficial Mexicana NOM-059SEMARNAT-2010, Protección ambiental - Especies nativas de México de flora y fauna silvestres - Categorías de riesgo y especificaciones para su inclusión, exclusión o cambio - Lista de especies en riesgo. Diario Oficial de la Federación. 2da Sección, 30 de diciembre de 2010.

SLPM. 2019. Herbario Isidro Palacios, Universidaad Autónoma de San Luis Potosí. http://slpm.uaslp.mx/ (accessed February 25, 2020)

Sosa V. 1988. Familia Staphyleaceae. Flora de Veracruz 57: 1-14.

Sosa V, De-Nova JA. 2012. Endemic angiosperm lineages in Mexico: hotspots for conservation. Acta Botanica Mexicana 100: 293-315. DOI: https://doi.org/10.21829/ abm100.2012.38

Sosa V, De-Nova JA, Vázquez-Cruz M. 2018. Evolutionary history of the flora of Mexico: dry forests cradles and museums of endemism. Journal of Systematics and Evolution 56: 523-536. DOI: https://doi.org/10.1111/ jse. 12416

Sosa V, Valdivieso IG. 2013. Familia Dioscoreaceae. Flora del Bajio y de Regiones Adyacentes 177: 1-38.

Spehn EM, Rudmann K, Korner C. 2010. Mountain Biodiversity and Global Change. GMBA-Diversitas Basel. ISBN: 9783605835236

Squeo FA, Cavieres LA, Arancio G, Novoa JE, Matthei O, Marticorena C, Rodríguez R, Arroyo MT, Muñoz M. 
1998. Biodiversidad de la Flora Vascular en la Región de Antofagasta, Chile. Revista Chilena de Historia Natural 71: $571-591$.

Steinmann V. 2005. Urticaceae. Flora del Bajío y de Regiones Adyacentes 134: 1-82.

Stevens WD, Morales JF. 2009. Apocynaceae. Flora Mesoamericana 1: 662-768. ISBN: 979-607-02-0901-7

ThePlantList. 2010. http://www.theplantlist.org/ (Accessed March 15, 2019).

Torres-Colín R, Gilberto PJ, De la Cruz LA, Ramírez MP, Gómez-Hinostrosa C, Bárcenas RT, Hernández HM. 2017. Flora vascular del municipio de Guadalcázar y zonas adyacentes, San Luis Potosí, México. Revista Mexicana de Biodiversidad 88: 524-554. DOI: http:// dx.doi.org/10.1016/j.rmb.2017.07.003

Torres JJ, Sierra RM. 2003. Las Áreas Naturales Protegidas del Estado de San Luis Potosí. San Luis Potosí: Secretaria de Ecología y Gestión Ambiental, Gobierno del Estado de San Luis Potosí.

Valencia-A S. 2004. Diversidad del género Quercus (Fagaceae) en México. Boletín de la Sociedad Botánica de México 75: 33-53. DOI: https://doi.org/10.17129/bot $\underline{\text { sci. } 1692}$

Villarreal H, Álvarez M, Córdoba S, Escobar F, Fagua G, Gast F, Mendoza H, Ospina M, Umaña AM. 2006. Manual de métodos para el desarrollo de inventarios de biodiversidad. Bogotá, Colombia: Instituto de

Editor de sección: Martha González Elizondo

Contribución de los autores: JIMT diseñó la investigación, realizó el trabajo de campo, la identificación taxonómica, el registro de datos, análisis, revisión de la nomenclatura, elaboración de mapas y estructuración del manuscrito. PCL participó en el diseño de la investigación, la supervisión y asesoría del proyecto, registro de datos, trabajo en campo, identificación taxonómica, la escritura y preparación de la versión final del manuscrito. RPM participó en el registro de los datos, trabajo en campo y la identificación taxonómica, la escritura y preparación de la versión final del manuscrito. JADN participó en el diseño de la investigación, la supervisión y asesoría del proyecto, revisión de la nomenclatura y análisis de datos, la escritura, generación de láminas y la preparación de la versión final del manuscrito. Todos los autores han contribuido sustancialmente con ideas y revisiones de las versiones previas del manuscrito.
Investigación de Recursos Biológicos Alexander von Humboldt. ISBN: 8151-32-5

Villaseñor JL. 2003. Diversidad y distribución de las Magnoliophyta de México. Interciencia 28: 160-167.

Villaseñor JL. 2004. Los géneros de plantas vasculares de la flora de México. Boletín de la Sociedad Botánica de México 75: 105-135. DOI: https://doi.org/10.17129/bot $\underline{\text { sci. } 1694}$

Villaseñor JL. 2010. El bosque húmedo de montaña en México y sus plantas vasculares: catálogo florísticotaxonómico. México, DF: Universidad Nacional Autónoma de México, Comisión Nacional para el Conocimiento y Uso de la Biodiversidad. ISBN: 9786070215575

Villaseñor JL. 2016. Checklist of the native vascular plants of Mexico. Revista Mexicana de Biodiversidad 3: 559-902. DOI: http://dx.doi.org/10.1016/j.rmb.2016.06. 017

Viviroli D, Weingartner R, Messerli B. 2003. Assessing the hydrological significance of the world's mountains. Mountain Research and Development 1: 32- 40. DOI: https://doi.org/10.1659/0276-4741(2003)023[0032:ATH $\underline{\mathrm{SOT}] 2.0 . \mathrm{CO} ; 2}$

Zavala-Chávez F, García-Sánchez F. 1999. Aspectos fisionómicos de la Sierra de Álvarez, San Luis Potosí, México. Revista Chapingo serie Ciencias Forestales y del Ambiente 1: 27-34. 
Apéndice 1. Lista de la flora vascular presente en la microcuenca del Cañón de los Chivos, sierra de Álvarez, San Luis Potosí, México. Todos los ejemplares citados se encuentran depositados en el herbario SLPM, excepto cuando se indique otro acrónimo. Forma de crecimiento y tipo de nutrición especial: Árbol (Ar); Arbusto (Ab); Epífita (Ep); Hierba (He); Acuatica (Ac); Parásita (Pa); Rupícola (Ru); Saprófita (Sa); Trepadora (Te). Recolectores: Pedro Castillo Lara (PCL); Felicidad García Sánchez (FGA); Raúl Puente Martínez (RPM); Jaime Iván Morales de la T. (JIMT); Edward Palmer (EP). Los ejemplares observados (Obs.) incluyen el número de catálogo de la base Naturalista CONABIO. Tipo de Vegetación: Bosque de encino (Be); Bosque afín a mesófilo de montaña (Bm); Bosque de encino-pino (Bep); Bosque de galería (Bg); Matorral submontano (Ms). Endemismo: Endémico de México (MX); Endémico de San Luis Potosí y regiones adyacentes (SLP). $\left(^{(}\right)$Especies no nativas de México. $(*)$ Nuevos registros para el estado.

\begin{tabular}{|c|c|c|c|c|}
\hline Clado/Familia/Especie & Recolector y no. de recolecta & F. de crec. y nutr. & T. de veg. & Endem. \\
\hline \multicolumn{5}{|l|}{ HELECHOS Y AFINES } \\
\hline \multicolumn{5}{|l|}{ Aspleniaceae } \\
\hline Asplenium monanthes $\mathrm{L}$. & JIMT 962, 977 & $\mathrm{He}$ & Bep & \\
\hline Asplenium praemorsum $\mathrm{Sw}$. & JIMT $604,704,863$ & $\mathrm{He}$ & $\mathrm{Be}, \mathrm{Bg}$ & \\
\hline Asplenium resiliens Kunze & JIMT 862 & $\mathrm{He}$ & $\mathrm{Bg}$ & \\
\hline \multicolumn{5}{|l|}{ Dryopteridaceae } \\
\hline Elaphoglossum muelleri (E. Fourn.) C. Chr. & JIMT 251, 590 & $\mathrm{He}$ & Bep, Bg & \\
\hline Phanerophlebia nobilis (Schltdl. \& Cham.) C. Presl & JIMT 17 & $\mathrm{He}$ & $\mathrm{Bm}$ & \\
\hline \multicolumn{5}{|l|}{ Equisetaceae } \\
\hline Equisetum hyemale $\mathrm{L}$. & JIMT 73, 421 & $\mathrm{He}$ & $\mathrm{Bg}$ & \\
\hline \multicolumn{5}{|l|}{ Ophioglossaceae } \\
\hline Botrychium schaffneri Underw. & JIMT 761 & $\mathrm{He}$ & $\mathrm{Be}, \mathrm{Bg}$ & \\
\hline Ophioglossum engelmannii Prantl & JIMT 899 & $\mathrm{He}$ & $\mathrm{Bg}$ & \\
\hline \multicolumn{5}{|l|}{ Polypodiaceae } \\
\hline Campyloneurum angustifolium (Sw.) Fée & JIMT 422B, 865 & Ep & $\mathrm{Be}, \mathrm{Bg}$ & \\
\hline Pecluma alfredii (Rosenst.) M.G. Price & JIMT 19, 703, 879, 924 & $\mathrm{He}$ & $\mathrm{Be}, \mathrm{Bep}, \mathrm{Bg}, \mathrm{Bm}$ & \\
\hline Phlebodium areolatum (Humb. \& Bonpl. ex Willd.) J. Sm. & JIMT 456, 544 & $\mathrm{He}$ & Bep, Bg & \\
\hline Phlebodium aureum (L.) J. Sm. & RPM 5186 & $\mathrm{He}$ & Bep & \\
\hline Pleopeltis macrocarpa (Bory ex Willd.) Kaulf. & JIMT $698,823,866$ & Ep & Be, Bep, Bg, Bm & \\
\hline Polypodium guttatum Maxon & JIMT $656,821,824$ & $\mathrm{He}$ & $\mathrm{Be}, \mathrm{Bep}$ & MX \\
\hline Polypodium madrense J. Sm. & JIMT 591, 655, 839 & $\mathrm{He}$ & Be, Bep & MX \\
\hline Polypodium polypodioides (L.) Watt & JIMT 697 & $\mathrm{He}$ & $\mathrm{Bg}$ & \\
\hline Polypodium subpetiolatum Hook. & JIMT $657,659,606$ & $\mathrm{He}$ & $\mathrm{Be}$ & \\
\hline Polypodium thyssanolepis A. Braun ex Klotzsch & JIMT 864 & $\mathrm{He}$ & $\mathrm{Bg}$ & \\
\hline \multicolumn{5}{|l|}{ Pteridaceae } \\
\hline Adiantum concinnum Humb. \& Bonpl. ex Willd. & JIMT, 197, 454, 716 & $\mathrm{He}$ & $\mathrm{Bg}, \mathrm{Bm}$ & \\
\hline Cheilanthes bonariensis (Willd.) Proctor & JIMT 705 & $\mathrm{He}$ & $\mathrm{Bg}$ & \\
\hline Cheilanthes lendigera (Cav.) Sw. & JIMT 822 & $\mathrm{He}$ & Bep & \\
\hline Cheilanthes notholaenoides (Desv.) Maxon ex Weath. & JIMT 658 & $\mathrm{He}$ & $\mathrm{Be}$ & \\
\hline Llavea cordifolia Lag. & JIMT 18 & $\mathrm{He}$ & $\mathrm{Bm}$ & \\
\hline Notholaena sulphurea (Cav.) J. Sm. & JIMT 869 & $\mathrm{He}$ & $\mathrm{Bg}$ & \\
\hline Pteris cretica $\mathrm{L}$. & JIMT 986 & $\mathrm{He}$ & $\mathrm{Be}$ & \\
\hline
\end{tabular}


Morales et al. / Botanical Sciences 98(4): 644-681. 2020

Clado/Familia/Especie

Selaginellaceae

Selaginella lepidophylla (Hook. \& Grev.) Spring

\section{GIMNOSPERMAS}

\section{Cupressaceae}

Juniperus flaccida Schltdl.

JIMT 59, 397, 843

Ar

Pinus arizonica Engelm.

Pinus cembroides Zucc.

Pinus devoniana Lindl.

Pinus greggii Engelm. ex Parl.

Pinus montezumae Lamb.

Pinus pseudostrobus Lindl.

Pinus strobiformis Engelm.

Pinus teocote Schied. ex Schltdl. \& Cham.

Zamiaceae

Ceratozamia zaragozae Medellín

\section{ANGIOSPERMAS}

\section{MANGNÓLIDAS}

\section{Lauraceae}

Cinnamomum pachypodum (Nees) Kosterm.

Litsea glaucescens Kunth

Litsea muelleri Rehder

Persea americana Mill.

Persea liebmannii $\mathrm{Mez}$

\section{Piperaceae}

Peperomia blanda (Jacq.) Kunth

Peperomia umbilicata Ruiz \& Pav.

Peperomia pringlei C. DC.

Peperomia quadrifolia (L.) Kunth

\section{MONOCOTILEDÓNEAS}

\section{Amaryllidaceae}

Allium glandulosum Link \& Otto

Sprekelia formosissima (L.) Herb.

Zephyranthes carinata Herb.

Zephyranthes concolor (Lindl.) Benth. \& Hook.f.

\section{Araceae}

Arisaema macrospathum Benth.

\section{Arecaceae}

Brahea dulcis (Kunth) Mart.

Recolector y no. de recolecta F. de crec. y nutr.

RPM 4868

$\mathrm{He}$

T. de veg.

Endem.

$\mathrm{Be}, \mathrm{Ms}$

JIMT 129 B, 377, 835
JIMT 376

JIMT 836

JIMT 834

JIMT 149, RPM 5212

JIMT 153 B

JIMT 126

JIMT 153 A, 378

PCL 1073

$\begin{array}{cc}\mathrm{Ar} & \text { Bep, Bg } \\ \mathrm{Ar} & \text { Bep } \\ \mathrm{Ar} & \text { Bep } \\ \mathrm{Ar} & \text { Bep } \\ \mathrm{Ar} & \text { Bep } \\ \mathrm{Ar} & \text { Bep } \\ \mathrm{Ar} & \text { Bep } \\ \mathrm{Ar} & \text { Bep }\end{array}$

$\mathrm{Ab}$

$\mathrm{Be}, \mathrm{Bg}$

SLP
JIMT 146, 189, $\quad$ Ar

JIMT 134

JIMT 570, 598

JIMT 86, 629

JIMT 227, 419, 856

JIMT 616

JIMT 225, RPM 5244

RPM 5166, 5202

JIMT 412, 460
$\mathrm{Ab}$

$\mathrm{Ab}$

Ar

Ar

$\mathrm{He}$

$\mathrm{He}$

$\mathrm{He}$

$\mathrm{He}$

$$
\mathrm{Be}, \mathrm{Bg}
$$

Be, Bep, Bg

$$
\text { Bep }
$$

$\mathrm{Bm}$

$$
\mathrm{Be}, \mathrm{Bg}
$$

$$
\begin{aligned}
& \mathrm{Be}, \mathrm{Bg}, \mathrm{Bm} \\
& \mathrm{Be}, \mathrm{Bep} \\
& \text { Bep }
\end{aligned}
$$

Be, Bep, Bg, Ms
JIMT, 145, 565

JIMT 150

JIMT 138

JIMT 1001
$\mathrm{He}$

$\mathrm{He}$

$\mathrm{He}$

$\mathrm{He}$

JIMT 232
$\mathrm{He}$

$\mathrm{Ar}$
MX

MX

MX
JIMT 1005

$\begin{array}{cc}\text { Be, Bep } & \\ \text { Be } & \text { MX } \\ \text { Be } & \\ \text { Be } & \text { MX }\end{array}$

Bm

MX 
Flora de la microcuenca del Cañón de Los Chivos

\begin{tabular}{|c|c|c|c|c|}
\hline Clado/Familia/Especie & Recolector y no. de recolecta & F. de crec. y nutr. & T. de veg. & Endem. \\
\hline Brahea moorei L.H.Bailey ex H.E.Moore & JIMT 1003 & $\mathrm{Ab}$ & $\mathrm{Be}, \mathrm{Bg}, \mathrm{Bm}$ & SLP \\
\hline \multicolumn{5}{|l|}{ Asparagaceae } \\
\hline Agave americana $\mathrm{L}$. & RPM 4859, 4896 & $\mathrm{Ab}$ & $\mathrm{Be}$ & \\
\hline Agave asperrima Jacobi & JIMT 643 & $\mathrm{Ab}$ & Ms & MX \\
\hline Agave filifera Salm-Dyck & JIMT 817 B & $\mathrm{Ab}$ & Bep & MX \\
\hline Agave garciae-mendozae Galván \& L.Hern. & JIMT 625 & $\mathrm{Ab}$ & $\mathrm{Be}, \mathrm{Bg}$ & SLP \\
\hline Agave mitis Mart. & JIMT 151 & $\mathrm{Ab}, \mathrm{Ru}$ & $\mathrm{Be}, \mathrm{Bg}, \mathrm{Bm}$ & SLP \\
\hline Agave salmiana Otto ex Salm-Dyck & JIMT 961 & $\mathrm{Ab}$ & $\mathrm{Be}$ & MX \\
\hline Agave schidigera Lem. & JIMT 817 A & $\mathrm{Ab}$ & Bep & MX \\
\hline Agave striata Zucc. & RPM 264, JIMT 529 B & $\mathrm{Ab}$ & $\mathrm{Be}$ & MX \\
\hline Dasylirion parryanum Trel. & JIMT 298 & $\mathrm{Ab}$ & Bep & SLP \\
\hline Echeandia durangensis (Greenm.) Cruden & JIMT 788 & $\mathrm{He}$ & $\mathrm{Be}$ & MX \\
\hline Echeandia flavescens (Schult. \& Schult.f.) Cruden & JIMT 533 & $\mathrm{He}$ & $\mathrm{Be}, \mathrm{Bg}, \mathrm{Ms}$ & \\
\hline Nolina orbicularis L. Hern. & JIMT 727 & $\mathrm{Ar}$ & Bep & MX \\
\hline Nolina parviflora (Kunth) Hemsl. & JIMT 990 & $\operatorname{Ar}$ & $\mathrm{Be}$ & $\mathrm{MX}$ \\
\hline Yucca potosina Rzed. & JIMT 110, 410, RPM 5201 & $\operatorname{Ar}$ & $\mathrm{Be}, \mathrm{Bg}, \mathrm{Ms}$ & SLP \\
\hline \multicolumn{5}{|l|}{ Bromeliaceae } \\
\hline Hechtia glomerata Zucc. & JIMT 131, 140 & $\mathrm{Ab}$ & $\mathrm{Bep}, \mathrm{Bg}, \mathrm{Bm}, \mathrm{Ms}$ & \\
\hline Tillandsia aguascalientensis C.S.Gardner & RPM 4790 B & Ep & $\mathrm{Be}$ & MX \\
\hline Tillandsia bartramii Elliott & JIMT 104 & Ep & $\mathrm{Bg}, \mathrm{Ms}$ & \\
\hline Tillandsia deppeana Steud. & JIMT 66, 435 & Ep & $\mathrm{Be}, \mathrm{Bg}, \mathrm{Bm}$ & \\
\hline Tillandsia erubescens Schltdl. & JIMT 512 & Ep & $\mathrm{Be}$ & MX \\
\hline Tillandsia inopinata Espejo, López-Ferr. \& W.Till & JIMT 757 & Ep & Ms & $\mathrm{MX}$ \\
\hline Tillandsia karwinskyana Schult. \& Schult.f. & JIMT 148, 624 & Ep & $\mathrm{Bg}, \mathrm{Bm}, \mathrm{Ms}$ & MX \\
\hline Tillandsia parryi Baker & JIMT 79, 997 & Ep & Bep, Bg & MX \\
\hline Tillandsia recurvata $(\mathrm{L}.) \mathrm{L}$. & JIMT 914 & Ep & Be, Bep, Ms & \\
\hline Tillandsia usneoides (L.) L. & JIMT 65, RPM 4796, 4798 B, 5237 & Ep & Be, Bep, Bg, Ms & \\
\hline \multicolumn{5}{|l|}{ Commelinaceae } \\
\hline Commelina coelestis Willd. & RPM 4855, 4860 & $\mathrm{He}$ & $\mathrm{Be}$ & \\
\hline Commelina dianthifolia Delile & RPM 4791 & $\mathrm{He}$ & Be, Bep & \\
\hline Commelina erecta $\mathrm{L}$. & JIMT 765 & $\mathrm{He}$ & $\mathrm{Be}$ & \\
\hline Tradescantia cirrifera Mart. & JIMT 556 & $\mathrm{He}$ & Bep & MX \\
\hline Tradescantia crassifolia Cav. & JIMT 444, 801, RPM 5559, 5560 & $\mathrm{He}$ & $\mathrm{Be}, \mathrm{Bep}, \mathrm{Bm}$ & \\
\hline${ }^{1}$ Tradescantia crassula Link \& Otto & JIMT 614 & $\mathrm{He}$ & $\mathrm{Be}, \mathrm{Bg}$ & \\
\hline \multicolumn{5}{|l|}{ Cyperaceae } \\
\hline Bulbostylis juncoides (Vahl) Kük. ex Herter & JIMT 681 & $\mathrm{He}$ & Bep & \\
\hline Cyperus spectabilis Link & JIMT 707 & $\mathrm{He}$ & $\mathrm{Bg}$ & \\
\hline Cyperus virens Michx. & JIMT 723 & $\mathrm{He}$ & Bep, Bg & \\
\hline
\end{tabular}


Morales et al. / Botanical Sciences 98(4): 644-681. 2020

\begin{tabular}{|c|c|c|c|c|}
\hline Clado/Familia/Especie & Recolector y no. de recolecta & F. de crec. y nutr. & T. de veg. & Endem. \\
\hline $\begin{array}{l}\text { Karinia mexicana (C.B.Clarke ex Britton) Reznicek \& } \\
\text { McVaugh }\end{array}$ & JIMT 553 & $\mathrm{He}$ & Bep & MX \\
\hline \multicolumn{5}{|l|}{ Dioscoreaceae } \\
\hline Dioscorea remotiflora Kunth & JIMT 621 & $\operatorname{Tr}$ & $\mathrm{Bg}$ & MX \\
\hline \multicolumn{5}{|l|}{ Iridaceae } \\
\hline \multicolumn{4}{|l|}{ Greenm. \& C.H.Thomps. } & MX \\
\hline Sisyrinchium cernuum (E.P.Bicknell) Kearney & JIMT 561 & $\mathrm{He}$ & Bep & \\
\hline Sisyrinchium scabrum Schltdl. \& Cham. & JIMT 773 & $\mathrm{He}$ & $\mathrm{Be}$ & \\
\hline Tigridia potosina López-Ferr. \& Espejo & JIMT 812 & $\mathrm{He}$ & $\mathrm{Be}$ & SLP \\
\hline Tigridia vanhouttei Roezl ex Van Houtte & Obs. 30699652 & $\mathrm{He}$ & Ms & MX \\
\hline \multicolumn{5}{|l|}{ Juncaceae } \\
\hline Juncus acuminatus Michx. & JIMT 922 & $\mathrm{He}$ & $\mathrm{Bg}$ & \\
\hline Juncus arcticus var. mexicanus Willd. & JIMT 712 & $\mathrm{He}$ & $\mathrm{Bg}$ & \\
\hline Juncus ebracteatus E.Mey. & JIMT 574 & $\mathrm{He}$ & Bep & \\
\hline \multicolumn{5}{|l|}{ Orchidaceae } \\
\hline Aulosepalum ramentaceum (Lindl.) Garay & JIMT 136, 426 & $\mathrm{He}$ & $\mathrm{Be}, \mathrm{Bep}, \mathrm{Bg}, \mathrm{Bm}$ & MX \\
\hline Bletia parkinsonii Hook. & JIMT 983 & $\mathrm{He}$ & $\mathrm{Be}, \mathrm{Ms}$ & MX \\
\hline Bletia purpurea (Lam.) A.DC. & JIMT 90 & $\mathrm{He}$ & $\mathrm{Bg}, \mathrm{Ms}$ & \\
\hline Bletia reflexa Lindl. & JIMT 351, 927 & $\mathrm{He}$ & $\mathrm{Bg}$ & \\
\hline Catasetum integerrimum Hook. & Obs. 30699924 & Ep & Ms & \\
\hline *Corallorhiza maculata Raf. & JIMT 585 & $\mathrm{He}, \mathrm{Sa}$ & Bep & \\
\hline Corallorhiza odontorhiza (Willd.) Nutt. & JIMT 830, 840, 901 & $\mathrm{He}, \mathrm{Sa}$ & Bep, Bg & \\
\hline Corallorhiza striata Lindl. & JIMT 770 & $\mathrm{He}, \mathrm{Sa}$ & $\mathrm{Be}$ & \\
\hline *Corallorhiza wisteriana Conrad & JIMT 84 & $\mathrm{He}, \mathrm{Sa}$ & $\mathrm{Bg}$ & \\
\hline Cranichis subumbellata A. Rich. \& Galeotti & JIMT 27 & $\mathrm{He}$ & $\mathrm{Bg}$ & MX \\
\hline Cyclopogon luteoalbus (A. Rich. \& Galeotti) Schltr. & JIMT 38, 467 & $\mathrm{He}$ & Bep, Bg & MX \\
\hline $\begin{array}{l}\text { Cyrtopodium macrobulbon (Lex.) G.A.Romero \& } \\
\text { Carnevali }\end{array}$ & JIMT 97 & $\mathrm{He}, \mathrm{Ru}$ & $\mathrm{Be}, \mathrm{Ms}$ & \\
\hline Dichromanthus cinnabarinus (Lex.) Garay & JIMT 217 & $\mathrm{He}$ & $\mathrm{Be}, \mathrm{Ms}$ & \\
\hline Epidendrum propinquum A. Rich. \& Galeotti & JIMT 36 B, 898 & Ep & $\mathrm{Bg}, \mathrm{Bm}$ & MX \\
\hline Epipactis gigantea Douglas ex Hook. & JIMT 92, 421 & $\mathrm{He}$ & $\mathrm{Bg}$ & \\
\hline *Funkiella tenella (L.O.Williams) Szlach. & JIMT 935 & $\mathrm{He}$ & Bep & MX \\
\hline Govenia lagenophora Lindl. & JIMT 1002, PCL 1066 & $\mathrm{He}$ & Bep, Bg & \\
\hline Govenia liliacea (Lex.) Lindl. & PCL 1029 & $\mathrm{He}$ & Be, Bep & \\
\hline Govenia purpusii Schltr. & JIMT 158 & $\mathrm{He}$ & $\mathrm{Be}$ & \\
\hline $\begin{array}{l}\text { Hexalectris grandiflora (A. Rich. \& Galeotti) } \\
\text { L.O.Williams }\end{array}$ & Obs. 30697936 & $\mathrm{He}, \mathrm{Sa}$ & Bep & \\
\hline *Hexalectris nitida L.O.Williams & JIMT 114 & $\mathrm{He}, \mathrm{Sa}$ & $\mathrm{Bg}, \mathrm{Ms}$ & \\
\hline Laelia anceps Lindl. & JIMT 15 & Ep & Bep, Bg, Bm, Ms & \\
\hline
\end{tabular}


Flora de la microcuenca del Cañón de Los Chivos

\begin{tabular}{|c|c|c|c|c|}
\hline Clado/Familia/Especie & Recolector y no. de recolecta & F. de crec. y nutr. & T. de veg. & Endem. \\
\hline Malaxis brachystachys (Rchb.f.) Kuntze & Obs. 30698959 & $\mathrm{He}$ & $\mathrm{Be}$ & \\
\hline Malaxis macrostachya (Lex.) Kuntze & JIMT 16 & $\mathrm{He}$ & Bep & \\
\hline Mesadenus polyanthus (Rchb.f.) Schltr. & JIMT 95 B & $\mathrm{He}$ & $\mathrm{Be}, \mathrm{Bm}, \mathrm{Ms}$ & \\
\hline Ponthieva ephippium Rchb.f. & JIMT 350 & $\mathrm{He}$ & $\mathrm{Bg}$ & \\
\hline Sarcoglottis schaffneri (Rchb.f.) Ames & JIMT 452 & $\mathrm{He}$ & $\mathrm{Be}, \mathrm{Bep}, \mathrm{Bg}, \mathrm{Bm}$ & \\
\hline $\begin{array}{l}\text { *Schiedeella crenulata (L.O.Williams) Espejo \& López- } \\
\text { Ferr. }\end{array}$ & JIMT 984 & $\mathrm{He}$ & Bep & MX \\
\hline Schiedeella llaveana (Lindl.) Schltr. & JIMT 95, 116 & $\mathrm{He}$ & $\mathrm{Be}, \mathrm{Bep}$ & \\
\hline Schiedeella nagelii (L.O.Williams) Garay & JIMT 11 & $\mathrm{He}$ & $\mathrm{Bg}, \mathrm{Bm}$ & SLP \\
\hline Schiedeella violacea (A. Rich. \& Galeotti) Garay & JIMT 54 & $\mathrm{He}$ & Bep, Be & \\
\hline Sotoa confusa (Garay) Salazar & JIMT 409 & $\mathrm{He}$ & $\mathrm{Be}$ & MX \\
\hline Triphora trianthophora (Sw.) Rydb. & JIMT 13 & $\mathrm{He}, \mathrm{Sa}$ & Be, Bep & \\
\hline \multicolumn{5}{|l|}{ Poaceae } \\
\hline Bothriochloa barbinodis (Lag.) Herter & JIMT 744 & $\mathrm{He}$ & Ms & \\
\hline Bothriochloa saccharoides (Sw.) Rydb. & JIMT 775 & $\mathrm{He}$ & $\mathrm{Be}$ & \\
\hline Bouteloua radicosa (E. Fourn.) Griffiths & JIMT 679 & $\mathrm{He}$ & Bep & MX \\
\hline Briza subaristata Lam. & JIMT 582, 801 & $\mathrm{He}$ & Be, Bep & \\
\hline Bromus anomalus E.Fourn. & JIMT 623 & $\mathrm{He}$ & $\mathrm{Bg}$ & \\
\hline Bromus carinatus Hook. \& Arn. & JIMT 626, 763 & $\mathrm{He}$ & $\mathrm{Be}, \mathrm{Bg}$ & \\
\hline Chondrosum hirsutum (Lag.) Sweet & JIMT 688 & $\mathrm{He}$ & Bep & \\
\hline${ }^{1}$ Cynodon dactylon (L.) Pers. & JIMT 583 & $\mathrm{He}$ & Bep & \\
\hline Eragrostis lugens Nees & JIMT 725 & $\mathrm{He}$ & $\mathrm{Bg}$ & \\
\hline Hilaria cenchroides Kunth & JIMT 689 & $\mathrm{He}$ & Bep & \\
\hline Lasiacis ruscifolia (Kunth) Hitchc. ex Chase & JIMT 23, RPM 5160 & $\mathrm{He}$ & $\mathrm{Be}, \mathrm{Bm}, \mathrm{Ms}$ & \\
\hline${ }^{1}$ Melinis repens (Willd.) Zizka & JIMT $347,400,562$ & $\mathrm{He}$ & $\mathrm{Bep}, \mathrm{Bg}, \mathrm{Ms}$ & \\
\hline Muhlenbergia macroura Hitchc. & JIMT 833 & $\mathrm{He}$ & Bep & \\
\hline Nassella leucotricha (Trin. \& Rupr.) R.W.Pohl & JIMT 680 & $\mathrm{He}$ & Bep & \\
\hline Oplismenus hirtellus (L.) P.Beauv. & JIMT 270, 608 & $\mathrm{He}$ & Be, Bep, Bg, Ms & \\
\hline Panicum bulbosum Kunth & JIMT 588 & $\mathrm{He}$ & Bep & \\
\hline Panicum decolorans Kunth & JIMT 714 & $\mathrm{He}$ & $\mathrm{Bg}$ & MX \\
\hline Panicum dichotomiflorum Michx. & JIMT 581, 641 & $\mathrm{He}$ & Bep, Ms & \\
\hline Panicum hallii Vasey & JIMT 921 & $\mathrm{He}$ & $\mathrm{Bg}$ & \\
\hline Panicum trichoides Sw. & JIMT 963 & $\mathrm{He}$ & Bep & \\
\hline Paspalum distichum $\mathrm{L}$. & JIMT 942 & $\mathrm{He}$ & Ms & \\
\hline Paspalum pubiflorum E.Fourn. & JIMT 711 & $\mathrm{He}$ & $\mathrm{Bg}$ & \\
\hline${ }^{1}$ Pennisetum glaucum (L.) R.Br. & JIMT 695 & $\mathrm{He}$ & $\mathrm{Bg}$ & \\
\hline Piptochaetium fimbriatum (Kunth) Hitchc. & JIMT 715 & $\mathrm{He}$ & $\mathrm{Bg}$ & \\
\hline${ }^{1}$ Poa annua $\mathrm{L}$. & JIMT 874,980 & $\mathrm{He}$ & $\mathrm{Be}, \mathrm{Bg}$ & \\
\hline Polypogon elongatus Kunth & JIMT 861 & $\mathrm{He}$ & $\mathrm{Bg}$ & \\
\hline
\end{tabular}


Morales et al. / Botanical Sciences 98(4): 644-681. 2020

\begin{tabular}{lllrr}
\hline Clado/Familia/Especie & Recolector y no. de recolecta & F. de crec. y nutr. & T. de veg. & Endem. \\
\hline Setaria grisebachii E.Fourn. & JIMT 748 & $\mathrm{He}$ & $\mathrm{Ms}$ & $\mathrm{Be}, \mathrm{Bg}$ \\
Sporobolus indicus (L.) R.Br. & JIMT 815, 923 & $\mathrm{He}$ & $\mathrm{Be}, \mathrm{Bg}$ & $\mathrm{MX}$
\end{tabular}

Smilacaceae

Smilax moranensis M.Martens \& Galeotti

JIMT 482, 525, 779, 994

$\operatorname{Tr}$

Be, Bep, Bm, Ms

Typhaceae

Typha domingensis Pers.

JIMT s.n.

$\mathrm{He}$

$\mathrm{Bg}$

Xanthorrhoeaceae

${ }^{1}$ Asphodelus fistulosus L.

RPM 5229, 407, 845

$\mathrm{He}$

Be, Bep, Bg, Ms

\section{EUDICOTILEDÓNEAS}

\section{Acanthaceae}

Dyschoriste schiedeana (Nees) Kuntze

JIMT 684

Justicia brandegeeana Wassh. \& L.B.Sm.

$\mathrm{He}$

Bep

Justicia leonardii Wassh.

Justicia tenera (Turrill) D.N. Gibson

Ruellia lactea Cav.

\section{Amaranthaceae}

Gomphrena serrata L.

Rhus aromatica Aiton

Toxicodendron radicans (L.) Kuntze

\section{Apiaceae}

Arracacia aegopodioides (Kunth) J.M. Coult. \& Rose

Cyclospermum leptophyllum (Pers.) Sprague

Eryngium hemsleyanum H.Wolff

Eryngium serratum Cav.

Sanicula liberta Cham. \& Schltdl.

Apocynaceae

Asclepias auriculata Kunth

Asclepias curassavica $\mathrm{L}$.

Asclepias linaria Cav.

Asclepias ovata M.Martens \& Galeotti

Asclepias pellucida E.Fourn.

JIMT 9, 35, 417, 858

JIMT 411, 883

JIMT 34, 896

$\mathrm{Ab}$

$\mathrm{Ab}$

JIMT 690

$\mathrm{Ab}$

$\mathrm{He}$

JIMT 528, 809

$\mathrm{Ar}$

Be, Bep

MX

JIMT 322

$\mathrm{He}$

JIMT 751

$\mathrm{He}$

JIMT 32, 895

$\mathrm{Ab}$

JIMT 191

$\operatorname{Ar}$

JIMT 169

JIMT 199, 851

JIMT 76, 427

JIMT 780, 798

JIMT 762, 981

JIMT 739

JIMT 567

JIMT 618, 768

JIMT 718

JIMT 135

JIMT 291, 472

JIMT 154, 685

$\mathrm{Be}, \mathrm{Bg}, \mathrm{Bm}, \mathrm{Ms}$

Ms MX

Bm, Ms

MX

$$
\text { Bep }
$$

Ms

Ms

$$
\mathrm{Be}, \mathrm{Bg}
$$

$\mathrm{Bg}, \mathrm{Ms}$

$\mathrm{Ab}$

$\mathrm{Ab}$

$\mathrm{Be}$

$\mathrm{Be}, \mathrm{Ms}$

MX

$\operatorname{Tr}$

$\mathrm{Be}, \mathrm{Bg}, \mathrm{Bm}$

$\mathrm{He}$

Be, Bep

$\mathrm{He}$

$\mathrm{He}$

$\mathrm{He}$

$\mathrm{Be}, \mathrm{Bg}$

$\mathrm{Be}, \mathrm{Ms}$

Be, Bep

MX

RPM 4879
$\mathrm{He}$

$\mathrm{Be}, \mathrm{Bg}$

$\mathrm{He}$

$\mathrm{He}$

$\mathrm{He}$

$\mathrm{He}$

$\mathrm{He}$

$$
\text { . }
$$

MX 
Flora de la microcuenca del Cañón de Los Chivos

\begin{tabular}{|c|c|c|c|c|}
\hline Clado/Familia/Especie & Recolector y no. de recolecta & F. de crec. y nutr. & T. de veg. & Endem. \\
\hline Asclepias similis Hemsl. & JIMT 778 & $\mathrm{He}$ & Be, Bep & \\
\hline Funastrum elegans (Decne.) Schltr. & JIMT 759 B, 559 B & $\operatorname{Tr}$ & Ms & MX \\
\hline Gomphocarpus fruticosus (L.) W.T.Aiton & $\begin{array}{l}\text { JIMT 451, 628, PCL 1074, RPM } \\
5169\end{array}$ & $\mathrm{He}$ & Bep, Bg & \\
\hline Mandevilla karwinskii (Müll.Arg.) Hemsl. & JIMT 638 & $\mathrm{He}$ & Ms & \\
\hline Marsdenia coulteri Hemsl. & JIMT 738 & $\operatorname{Tr}$ & Ms & MX \\
\hline Matelea pilosa (Benth.) Woodson & PCL 1099 & $\operatorname{Tr}$ & Ms & MX \\
\hline Matelea reticulata (Engelm. ex A.Gray) Woodson & JIMT 803, PCL 1131 & $\operatorname{Tr}$ & $\mathrm{Be}, \mathrm{Bg}, \mathrm{Ms}$ & \\
\hline Metastelma angustifolium Turcz. & JIMT 224, 605, 637, & $\operatorname{Tr}$ & $\mathrm{Be}, \mathrm{Ms}$ & MX \\
\hline Metastelma schlechtendalii Decne. & $4856 \mathrm{RPM}$ & $\operatorname{Tr}$ & $\mathrm{Be}$ & \\
\hline \multicolumn{5}{|l|}{ Aquifoliaceae } \\
\hline Ilex brandegeeana Loes. & JIMT $155,423,630$ & $\mathrm{Ar}$ & $\mathrm{Be}, \mathrm{Bg}$ & \\
\hline \multicolumn{5}{|l|}{ Asteraceae } \\
\hline Acourtia coulteri (A.Gray) Reveal \& R.M.King & PCL 1125 & $\mathrm{He}$ & $\mathrm{Bg}$ & \\
\hline Ageratina calaminthaefolia (Kunth) R.M.King \& H.Rob. & JIMT 720 & $\mathrm{Ab}$ & $\mathrm{Bg}$ & MX \\
\hline Ageratina glabrata (Kunth) R.M.King \& H.Rob. & PCL 1135, JIMT 818, 919 & $\mathrm{Ab}$ & Bep, Bg & MX \\
\hline $\begin{array}{l}\text { Ageratina petiolaris (Moc. \& Sessé ex DC.) R.M.King \& } \\
\text { H.Rob. }\end{array}$ & JIMT 399, 969 & $\mathrm{Ab}$ & $\mathrm{Be}, \mathrm{Ms}$ & MX \\
\hline Ageratina rhomboidea (Kunth) R.M.King \& H.Rob. & JIMT 875 & $\mathrm{Ab}$ & $\mathrm{Be}$ & MX \\
\hline $\begin{array}{l}\text { Ageratina schaffneri (Sch.Bip. ex B.L. Rob.) R.M.King \& } \\
\text { H.Rob. }\end{array}$ & JIMT 371, 391 & $\mathrm{He}$ & Bep & \\
\hline Aster subulatus (Michx.) Hort. ex Michx. & JIMT 844,860 & $\mathrm{He}$ & $\mathrm{Bg}, \mathrm{Ms}$ & \\
\hline Baccharis heterophylla Kunth & PCL 1124, JIMT 458 & $\mathrm{Ab}$ & Bep, Bg & \\
\hline Baccharis salicina Torr. \& A.Gray & JIMT 195 & $\mathrm{He}$ & $\mathrm{Be}, \mathrm{Bg}$ & \\
\hline Baccharis sulcata DC. & JIMT 829 & $\mathrm{He}$ & Bep & \\
\hline Barkleyanthus salicifolius (Kunth) H. Rob. \& Brettell & JIMT 474 & $\mathrm{Ab}$ & Ms & \\
\hline Bidens angustissima Kunth & JIMT 578 & $\mathrm{He}$ & Bep & MX \\
\hline Bidens ostruthioides (DC.) Sch.Bip. & JIMT 862 & $\mathrm{He}$ & Ms & \\
\hline Bidens pilosa $\mathrm{L}$. & JIMT 910 & $\mathrm{He}$ & Bep & \\
\hline Bidens triplinervia Kunth & JIMT 973 & $\mathrm{He}$ & $\mathrm{Be}$ & \\
\hline Calyptocarpus vialis Less. & JIMT 731 & $\mathrm{He}$ & Ms & \\
\hline Carphochaete grahamii A.Gray & JIMT $827,841,912$ & $\mathrm{He}$ & Be, Bep & MX \\
\hline Cirsium ehrenbergii Sch.Bip. & JIMT 396 & $\mathrm{He}$ & $\mathrm{Be}$ & MX \\
\hline Cirsium mexicanum DC. & JIMT 360 & $\mathrm{He}$ & Be, Bep & \\
\hline Cosmos atrosanguineus (Hook.) Voss & JIMT 14 & $\mathrm{He}$ & Bep, Bg & SLP \\
\hline Cosmos bipinnatus Cav. & JIMT 361 & $\mathrm{He}$ & $\mathrm{Be}$ & \\
\hline Cosmos diversifolius Otto ex Knowles \& Westc. & JIMT 800 & $\mathrm{He}$ & Be, Bep & \\
\hline Cosmos pacificus Melchert & JIMT 911 & $\mathrm{He}$ & Be, Bep & MX \\
\hline Dahlia coccinea Cav. & JIMT 392, 609, PCL 1130 & $\mathrm{He}$ & $\mathrm{Be}, \mathrm{Bg}$ & \\
\hline Erigeron karvinskianus DC. & JIMT 321, 430, 486, 631, 709, 859 & $\mathrm{He}$ & $\mathrm{Bg}, \mathrm{Ms}$ & \\
\hline
\end{tabular}


Morales et al. / Botanical Sciences 98(4): 644-681. 2020

\begin{tabular}{|c|c|c|c|c|}
\hline Clado/Familia/Especie & Recolector y no. de recolecta & F. de crec. y nutr. & T. de veg. & Endem. \\
\hline Erigeron pubescens Kunth & JIMT 772 & $\mathrm{He}$ & $\mathrm{Be}$ & \\
\hline Fleischmannia pycnocephala (Less.) R.M.King \& H.Rob. & JIMT 568, 782 & $\mathrm{He}$ & Be, Bep & \\
\hline Grindelia inuloides Willd. & JIMT 750 & $\mathrm{He}$ & $\mathrm{Be}, \mathrm{Ms}$ & MX \\
\hline Gutierrezia texana var. glutinosa (S.Schauer) M.A.Lane & $\begin{array}{l}\text { JIMT 732, 848, 887, 903, 917, 956, } \\
959\end{array}$ & $\mathrm{He}$ & $\mathrm{Bep}, \mathrm{Bg}, \mathrm{Ms}$ & \\
\hline Helenium mexicanum Kunth & JIMT $60,665,949,952$ & $\mathrm{He}$ & Be, Bep, Ms & \\
\hline Hieracium pringlei A.Gray & JIMT 560 & $\mathrm{He}$ & $\mathrm{Bep}, \mathrm{Bg}$ & \\
\hline Iostephane heterophylla (Cav.) Benth. & JIMT 767 & $\mathrm{He}$ & $\mathrm{Be}$ & MX \\
\hline Jefea lantanifolia (Schauer) Strother & PCL 1076 & $\mathrm{He}$ & $\mathrm{Bg}$ & MX \\
\hline Perymenium buphthalmoides DC. & JIMT 628 & $\mathrm{He}$ & Bep & MX \\
\hline Perymenium mendezii DC. & JIMT 577 & $\mathrm{Ab}$ & Bep & MX \\
\hline Piqueria trinervia Cav. & $\begin{array}{l}\text { JIMT 40, 667, 872, 877, 907, 931, } \\
957\end{array}$ & $\mathrm{He}$ & $\mathrm{Be}, \mathrm{Bep}, \mathrm{Ms}$ & \\
\hline Porophyllum linaria (Cav.) DC. & JIMT 890 & $\mathrm{He}$ & Ms & MX \\
\hline Psacalium peltatum (Kunth) Cass. & JIMT 870 & $\mathrm{He}$ & $\mathrm{Be}, \mathrm{Bg}$ & MX \\
\hline Psacalium sinuatum (Cerv.) H. Rob. \& Brettell & JIMT 555, PCL 1115 & $\mathrm{He}$ & Bep, Bg & MX \\
\hline Pseudognaphalium roseum (Kunth) Anderb. & JIMT 819 & $\mathrm{He}$ & Bep & \\
\hline Pseudognaphalium stramineum (Kunth) Anderb. & JIMT 509, 600 & $\mathrm{He}$ & Be, Bep & \\
\hline Roldana platanifolia (Benth.) H. Rob. \& Brettell & JIMT 28, 868, 918 & $\mathrm{He}$ & $\mathrm{Bg}$ & MX \\
\hline Rumfordia alcortae Rzed. & JIMT 25, 601 & $\mathrm{He}$ & $\mathrm{Be}$ & SLP \\
\hline $\begin{array}{l}\text { Sclerocarpus uniserialis (Hook.) Benth. \& Hook.f. ex } \\
\text { Hemsl. }\end{array}$ & JIMT 607, RPM 5181 & $\mathrm{He}$ & Be, Bep & \\
\hline Senecio aschenbornianus S.Schauer & $\begin{array}{l}\text { JIMT 47, 403, 837, 850, 871, 975, } \\
\text { RPM 5211, } 5232\end{array}$ & $\mathrm{Ab}$ & Be, Bep, Bg, Ms & \\
\hline Senecio praecox (Cav.) DC. & $\begin{array}{l}\text { JIMT } 138 \text { B, 398, 406, RPM } 4795 \text {, } \\
4895\end{array}$ & $\mathrm{Ab}$ & $\mathrm{Be}, \mathrm{Bg}, \mathrm{Ms}$ & MX \\
\hline${ }^{1}$ Sonchus oleraceus (L.) L. & JIMT 36 & $\mathrm{He}$ & $\mathrm{Bg}$ & \\
\hline Stevia elatior Kunth & JIMT 545 & $\mathrm{He}$ & Bep & \\
\hline Stevia ovata Willd. & JIMT 547, 573, 597, 793, 913 & $\mathrm{Ab}$ & Be, Bep & \\
\hline Stevia serrata Cav. & JIMT 676 & $\mathrm{He}$ & Bep & \\
\hline Stevia stricta Hornem. & JIMT 546, PCL 1123 & $\mathrm{Ab}$ & Bep, Bg & \\
\hline Stevia viscida Kunth & RPM 5179, 5207, 5216 & $\mathrm{He}$ & Bep & \\
\hline Tagetes lucida Cav. & JIMT 355 & $\mathrm{He}$ & $\mathrm{Be}$ & \\
\hline Tagetes lunulata Ortega & JIMT 261 & $\mathrm{He}$ & Ms & MX \\
\hline Tagetes micrantha Cav. & RPM 5224, 5554 & $\mathrm{He}$ & Be, Bep & \\
\hline Tagetes parryi A.Gray & JIMT 854 & $\mathrm{He}$ & Ms & SLP \\
\hline${ }^{1}$ Taraxacum campylodes G.E.Haglund & JIMT 495 & $\mathrm{He}$ & $\mathrm{Be}$ & \\
\hline Tithonia tubaeformis (Jacq.) Cass. & JIMT 814 & $\mathrm{He}$ & $\mathrm{Be}, \mathrm{Bg}, \mathrm{Ms}$ & \\
\hline Verbesina sororia A.Gray & JIMT 799, 810 & $\mathrm{Ab}$ & $\mathrm{Be}$ & SLP \\
\hline Vernonia alamanii DC. & JIMT 652, 867, PCL 1128 & $\mathrm{He}$ & $\mathrm{Be}, \mathrm{Bg}$ & MX \\
\hline
\end{tabular}


Flora de la microcuenca del Cañón de Los Chivos

\begin{tabular}{|c|c|c|c|c|}
\hline Clado/Familia/Especie & Recolector y no. de recolecta & F. de crec. y nutr. & T. de veg. & Endem. \\
\hline Vernonia greggii (A.Gray) B.L.Turner & JIMT 954 & $\mathrm{Ab}$ & Ms & MX \\
\hline Viguiera trachyphylla S.F.Blake & JIMT 535 & $\mathrm{Ab}$ & Ms & MX \\
\hline Zinnia acerosa (DC.) A.Gray & JIMT 832 & $\mathrm{He}$ & Bep & \\
\hline Zinnia peruviana (L.) L. & JIMT 332 & $\mathrm{He}$ & $\mathrm{Bg}, \mathrm{Ms}$ & \\
\hline \multicolumn{5}{|l|}{ Begoniaceae } \\
\hline Begonia gracilis Kunth & RPM 5167, PCL 1126, JIMT 303 & $\mathrm{He}$ & Bep, Bg, Ms & \\
\hline \multicolumn{5}{|l|}{ Berberidaceae } \\
\hline Berberis aristata DC. & JIMT 77, 424 & $\mathrm{Ab}$ & $\mathrm{Be}, \mathrm{Bg}, \mathrm{Ms}$ & $\mathrm{MX}$ \\
\hline \multicolumn{5}{|l|}{ Betulaceae } \\
\hline Alnus acuminata subsp. arguta (Schltdl.) Furlow & JIMT 185 & $\mathrm{Ar}$ & $\mathrm{Bg}$ & \\
\hline \multicolumn{5}{|l|}{ Bignoniaceae } \\
\hline Tecoma stans (L.) Juss. ex Kunth & JIMT 201, 478 & $\mathrm{Ab}$ & $\mathrm{Be}, \mathrm{Ms}$ & \\
\hline \multicolumn{5}{|l|}{ Boraginaceae } \\
\hline Antiphytum heliotropioides A.DC. & JIMT 617 & $\mathrm{He}$ & $\mathrm{Bg}$ & \\
\hline Lithospermum pringlei I.M. Johnst. & JIMT 796 & $\mathrm{He}$ & $\mathrm{Be}$ & \\
\hline Lithospermum viride Greene & PCL 1071 & $\mathrm{He}$ & $\mathrm{Bg}$ & \\
\hline Nama biflora Choisy & JIMT 946 & $\mathrm{He}$ & Ms & MX \\
\hline Nama origanifolia Kunth & JIMT 471 & $\mathrm{He}$ & $\mathrm{Bg}, \mathrm{Ms}$ & MX \\
\hline Nama sericea Willd. ex Roem. \& Schult. & JIMT 236, 266, 900, RPM 5177 & $\mathrm{He}$ & $\mathrm{Bep}, \mathrm{Bg}, \mathrm{Bm}, \mathrm{Ms}$ & MX \\
\hline Tournefortia densiflora M.Martens \& Galeotti & JIMT 937 & $\mathrm{Ab}$ & Ms & \\
\hline \multicolumn{5}{|l|}{ Brassicaceae } \\
\hline${ }^{1}$ Cardamine hirsuta $\mathrm{L}$. & RPM 5234 & $\mathrm{He}$ & Bep & \\
\hline Lepidium virginicum $\mathrm{L}$. & JIMT 944 & $\mathrm{He}$ & Ms & \\
\hline${ }^{1}$ Nasturtium officinale $\mathrm{R} . \mathrm{Br}$. & JIMT 988 & $\mathrm{He}, \mathrm{Ac}$ & $\mathrm{Be}, \mathrm{Bg}$ & \\
\hline \multicolumn{5}{|l|}{ Burseraceae } \\
\hline Bursera fagaroides (Kunth) Engl. & JIMT 352, 737 & $\mathrm{Ab}$ & Ms & \\
\hline \multicolumn{5}{|l|}{ Cactaceae } \\
\hline Cylindropuntia imbricata (Haw.) F.M.Knuth & JIMT 475 & $\mathrm{Ab}$ & Ms & \\
\hline Cylindropuntia leptocaulis (DC.) F.M.Knuth & JIMT 477 & $\mathrm{Ab}$ & Ms & \\
\hline Echinocereus pentalophus (DC.) Lem. & JIMT 1007 & $\mathrm{Ab}$ & $\mathrm{Be}$ & \\
\hline Echinocereus polyacanthus var. densus (Regel) & Obs. 30733907 & $\mathrm{Ab}$ & Bep, Bg & \\
\hline \multicolumn{5}{|l|}{ N.P.Taylor. } \\
\hline Ferocactus echidne (DC.) Britton \& Rose & RPM 4797 & $\mathrm{Ab}$ & $\mathrm{Be}$ & \\
\hline Ferocactus glaucescens (DC.) Britton \& Rose & Obs. 30699474 & $\mathrm{Ab}$ & Ms & MX \\
\hline Ferocactus histrix (DC.) G.E.Linds. & JIMT 476 & $\mathrm{Ab}$ & Ms & MX \\
\hline Mammillaria densispina (J.M. Coult.) Orcutt & JIMT 364 & $\mathrm{Ab}$ & Bep & $\mathrm{MX}$ \\
\hline Mammillaria erythrosperma Boed. & JIMT 108 & $\mathrm{He}$ & $\mathrm{Bg}, \mathrm{Bm}$ & SLP \\
\hline Mammillaria schiedeana Ehrenb. ex Schltdl. & JIMT 951 & $\mathrm{Ab}$ & Ms & SLP \\
\hline Mammillaria uncinata Zucc. ex Pfeiff. & JIMT 363 & $\mathrm{Ab}$ & Bep & MX \\
\hline
\end{tabular}


Morales et al. / Botanical Sciences 98(4): 644-681. 2020

\begin{tabular}{|c|c|c|c|c|}
\hline Clado/Familia/Especie & Recolector y no. de recolecta & F. de crec. y nutr. & T. de veg. & Endem. \\
\hline Myrtillocactus geometrizans (Mart. ex Pfeiff.) Console & JIMT 536 & $\mathrm{Ab}$ & Ms & MX \\
\hline Opuntia leucotricha DC. & Obs. 9342234 & $\mathrm{Ab}$ & $\mathrm{Be}$ & MX \\
\hline Opuntia megarrhiza Rose & EP 607 (US) & $\mathrm{Ab}$ & $\mathrm{Be}$ & SLP \\
\hline Opuntia neochrysacantha Bravo & JIMT 895 B & $\mathrm{Ab}$ & Ms & MX \\
\hline $\begin{array}{l}\text { Opuntia pachyrrhiza H.M. Hern., Gomez-Hin. \& } \\
\text { Barcenas }\end{array}$ & RPM 469, 4887 & $\mathrm{Ab}$ & $\mathrm{Be}$ & MX \\
\hline Opuntia robusta J.C. Wendl. & Obs. 30700229 & $\mathrm{Ab}$ & $\mathrm{Be}$ & \\
\hline Selenicereus spinulosus (DC.) Britton \& Rose & JIMT 538 & $\operatorname{Tr}$ & $\mathrm{Be}, \mathrm{Bg}, \mathrm{Bm}, \mathrm{Ms}$ & \\
\hline $\begin{array}{l}\text { Stenocereus queretaroensis (F.A.C. Weber ex Mathes.) } \\
\text { Buxb. }\end{array}$ & JIMT 99 & $\mathrm{Ab}$ & Ms & \\
\hline \multicolumn{5}{|l|}{ Campanulaceae } \\
\hline Diastatea micrantha (Kunth) McVaugh & JIMT 904 & $\mathrm{He}$ & Bep, Ms & \\
\hline Lobelia gruina Cav. & JIMT 62, 354, 790, 932, 970 & $\mathrm{He}$ & Be, Bep, Ms & $\mathrm{MX}$ \\
\hline Lobelia laxiflora Kunth & JIMT 72, 422, & $\mathrm{He}$ & $\mathrm{Bg}$ & \\
\hline \multicolumn{5}{|l|}{ Cannabaceae } \\
\hline Celtis pallida Torr. & JIMT 247, 743 & $\mathrm{Ab}$ & $\mathrm{Bg}, \mathrm{Ms}$ & \\
\hline \multicolumn{5}{|l|}{ Caprifoliaceae } \\
\hline Valeriana albonervata B.L. Rob. & JIMT 141, 175, 878 & $\mathrm{He}$ & $\mathrm{Be}$ & SLP \\
\hline \multicolumn{5}{|l|}{ Caryophyllaceae } \\
\hline Arenaria lycopodioides Willd. ex Schltdl. & FGS 6660, JIMT s.n. & $\mathrm{He}$ & $\mathrm{Be}$ & \\
\hline Drymaria villosa Schltdl. \& Cham. & JIMT 974 & $\mathrm{He}$ & $\mathrm{Be}$ & \\
\hline Silene laciniata Cav. & JIMT 802 & $\mathrm{He}$ & $\mathrm{Be}$ & \\
\hline \multicolumn{5}{|l|}{ Ceratophyllaceae } \\
\hline Ceratophyllum demersum $\mathrm{L}$. & JIMT1006 & $\mathrm{He}, \mathrm{Ac}$ & $\mathrm{Bg}$ & \\
\hline \multicolumn{5}{|l|}{ Cistaceae } \\
\hline Helianthemum patens Hemsl. & JIMT 503, 831 & $\mathrm{He}$ & Be, Bep & MX \\
\hline \multicolumn{5}{|l|}{ Cleomaceae } \\
\hline Polanisia uniglandulosa (Cav.) DC. & JIMT 759 & $\mathrm{He}$ & Ms & \\
\hline \multicolumn{5}{|l|}{ Convolvulaceae } \\
\hline Cuscuta corymbosa Ruiz \& Pav. & JIMT $633,880,886$ & $\operatorname{Tr}$ & Ms & \\
\hline Cuscuta tinctoria Mart. ex Engelm. & JIMT 48, 603, 953 & $\operatorname{Tr}$ & $\mathrm{Be}, \mathrm{Ms}$ & \\
\hline Dichondra argentea Humb. \& Bonpl. ex Willd. & JIMT 551, 708, 889 & $\mathrm{He}$ & $\mathrm{Bep}, \mathrm{Bg}, \mathrm{Ms}$ & \\
\hline Evolvulus alsinoides (L.) L. & JIMT 532, 742 & $\mathrm{He}$ & Ms & \\
\hline Evolvulus sericeus $\mathrm{Sw}$. & JIMT 550 & $\mathrm{He}$ & Bep & \\
\hline Ipomoea orizabensis (G. Pelletan) Ledeb. ex Steud. & JIMT 222, 647, 719, 998 & $\operatorname{Tr}$ & $\mathrm{Be}, \mathrm{Bg}, \mathrm{Ms}$ & \\
\hline \multicolumn{5}{|l|}{ Cornaceae } \\
\hline Cornus disciflora Moc. \& Sessé ex DC. & JIMT 56, 383 & $\mathrm{Ar}$ & Be, Bep & \\
\hline \multicolumn{5}{|l|}{ Crassulaceae } \\
\hline Echeveria agavoides Lem. & JIMT 78, 389 & $\mathrm{He}$ & Bep, Bg & MX \\
\hline Echeveria bifida Schltdl. & JIMT 170 & $\mathrm{He}$ & $\mathrm{Be}$ & MX \\
\hline
\end{tabular}


Flora de la microcuenca del Cañón de Los Chivos

\begin{tabular}{|c|c|c|c|c|}
\hline Clado/Familia/Especie & Recolector y no. de recolecta & F. de crec. y nutr. & T. de veg. & Endem. \\
\hline Echeveria elegans Rose & JIMT 432 & $\mathrm{He}$ & $\mathrm{Be}, \mathrm{Bg}, \mathrm{Ms}$ & MX \\
\hline Echeveria humilis Rose & JIMT 78 B, 458 & $\mathrm{He}$ & Bep, Bg & SLP \\
\hline Echeveria lutea Rose & JIMT 642, RPM 4788, 4864 & $\mathrm{He}$ & $\mathrm{Be}, \mathrm{Bg}$ & SLP \\
\hline Echeveria mucronata Schltdl. & JIMT 571 & $\mathrm{He}$ & Bep & MX \\
\hline Echeveria subrigida (B.L. Rob. \& Seaton) Rose & JIMT 860 & $\mathrm{He}$ & $\mathrm{Be}$ & MX \\
\hline Sedum calcicola B.L. Rob. \& Greenm. & JIMT 238 & $\mathrm{He}$ & $\mathrm{Be}, \mathrm{Ms}$ & MX \\
\hline Sedum clausenii Pérez-Calix & JIMT 434 & $\mathrm{He}$ & Be, Bep & MX \\
\hline Sedum ebracteatum Moc. \& Sessé ex DC. & JIMT 469, 936, 979 & $\mathrm{He}$ & $\mathrm{Bg}, \mathrm{Ms}$ & \\
\hline Sedum moranense Kunth & JIMT 173, 558 & $\mathrm{He}$ & Be, Bep & \\
\hline Sedum palmeri S. Watson & JIMT 439 & $\mathrm{He}$ & $\mathrm{Be}, \mathrm{Bg}, \mathrm{Bm}$ & MX \\
\hline Sedum retusum Hemsl. & JIMT 805, RPM 5205, 5245 & $\mathrm{He}$ & $\mathrm{Be}$ & MX \\
\hline Villadia patula Moran \& C.H. Uhl & RPM 4792 & $\mathrm{He}$ & $\mathrm{Be}$ & SLP \\
\hline Villadia recurva Moran, Kimnach \& C.H. Uhl & RPM 5204 & $\mathrm{He}$ & Bep & MX \\
\hline \multicolumn{5}{|l|}{ Cucurbitaceae } \\
\hline Microsechium palmatum (Ser.) Cogn. & JIMT 353,783 & $\operatorname{Tr}$ & $\mathrm{Be}$ & \\
\hline \multicolumn{5}{|l|}{ Ericaceae } \\
\hline Arbutus tessellata P.D.Sørensen & JIMT 123, 386 & $\mathrm{Ar}$ & Bep & MX \\
\hline Arbutus xalapensis Kunth & JIMT 365 & $\mathrm{Ar}$ & Be, Bep & \\
\hline Arctostaphylos pungens Kunth & PCL 1140, JIMT 369, 826 & $\mathrm{Ab}$ & Bep & \\
\hline Chimaphila maculata (L.) Pursh & JIMT 993 & $\mathrm{He}$ & Bep & \\
\hline Comarostaphylis polifolia (Kunth) Zucc. ex Klotzsch & PCL 1145, RPM 4798 & $\mathrm{Ab}$ & Be, Bep & MX \\
\hline Gaultheria erecta Vent. & JIMT 991 & $\mathrm{Ab}$ & Bep & \\
\hline Lyonia squamulosa M. Martens \& Galeotti & JIMT 572, 828, 996 & $\mathrm{Ab}$ & Bep & \\
\hline Polycodium kunthianum (Klotzsch) C.B. Rob. & JIMT 992 & $\mathrm{Ab}$ & Bep & $\mathrm{MX}$ \\
\hline Vaccinium confertum Kunth & FGS 2262 & $\mathrm{Ab}$ & Bep & \\
\hline \multicolumn{5}{|l|}{ Euphorbiaceae } \\
\hline Acalypha phleoides Cav. & JIMT 218 & $\mathrm{He}$ & $\mathrm{Be}$ & \\
\hline Acalypha subviscida S.Watson & JIMT 706, 749 & $\mathrm{He}$ & $\mathrm{Bg}, \mathrm{Ms}$ & \\
\hline Bernardia albida Lundell & JIMT 635,885 & $\mathrm{Ar}$ & Ms & MX \\
\hline Bernardia mexicana (Hook. \& Arn.) Müll.Arg. & JIMT 1004 & $\mathrm{Ab}$ & $\mathrm{Bg}$ & \\
\hline Croton ciliatoglandulifer Ortega & $\begin{array}{l}\text { JIMT } 153,250,754,755,934, \\
\text { RPM 5189, } 5557\end{array}$ & $\mathrm{Ab}$ & Be, Bep, Bg, Ms & \\
\hline Croton disjunctus V.W.Steinm. & JIMT 664 & $\mathrm{Ab}$ & $\mathrm{Be}$ & $\mathrm{MX}$ \\
\hline Euphorbia anychioides Boiss. & JIMT 569, 691 & $\mathrm{He}$ & Bep & \\
\hline Euphorbia cymosa Poir. & JIMT 96, 453, 897 & $\mathrm{Ab}$ & $\mathrm{Be}, \mathrm{Bg}$ & \\
\hline Euphorbia macropus (Klotzsch \& Garcke) Boiss. & JIMT 620 & $\mathrm{He}$ & $\mathrm{Bg}, \mathrm{Bm}$ & \\
\hline Euphorbia potosina Fernald & JIMT 675 & $\mathrm{He}$ & Bep & MX \\
\hline Euphorbia radians Benth. & JIMT 455 & $\mathrm{He}$ & Bep & \\
\hline Euphorbia sphaerorhiza Benth. & JIMT 554 & $\mathrm{He}$ & Bep & MX \\
\hline
\end{tabular}


Morales et al. / Botanical Sciences 98(4): 644-681. 2020

\begin{tabular}{|c|c|c|c|c|}
\hline Clado/Familia/Especie & Recolector y no. de recolecta & F. de crec. y nutr. & T. de veg. & Endem. \\
\hline Sebastiania pavoniana (Müll.Arg.) Müll.Arg. & JIMT 102, 491 & $\mathrm{Ar}$ & Ms & \\
\hline Stillingia zelayensis (Kunth) Müll.Arg. & JIMT 615 & $\mathrm{Ab}$ & $\mathrm{Bg}$ & \\
\hline \multicolumn{5}{|l|}{ Fabaceae } \\
\hline Bauhinia coulteri J.F.Macbr. & $\begin{array}{l}\text { RPM 4863, 5176, 5555, JIMT 646, } \\
842\end{array}$ & $\mathrm{Ab}$ & Be, Bep, Ms & MX \\
\hline Bauhinia macranthera Hemsl. & JIMT 152 & $\mathrm{Ab}$ & $\mathrm{Be}$ & MX \\
\hline Brongniartia discolor Brandegee & JIMT 745 & $\mathrm{Ab}$ & Ms & SLP \\
\hline Cercis canadensis $\mathrm{L}$. & JIMT 441 & $\mathrm{Ar}$ & $\begin{array}{c}\mathrm{Be}, \mathrm{Bg}, \mathrm{Bm}, \mathrm{Bm}, \\
\mathrm{Ms}\end{array}$ & \\
\hline Chamaecrista glandulosa (L.) Greene & JIMT 674 & $\mathrm{Ab}$ & Bep & \\
\hline Cologania broussonetii (Balb.) DC. & JIMT 661 & $\operatorname{Tr}$ & $\mathrm{Be}$ & \\
\hline Crotalaria mollicula Kunth & JIMT 559 & $\mathrm{He}$ & Bep & \\
\hline Crotalaria sagittalis $\mathrm{L}$. & JIMT 678 & $\mathrm{He}$ & Bep & \\
\hline Dalea bicolor Willd. & JIMT 315,640 & $\mathrm{Ab}$ & Ms & \\
\hline Dalea lutea (Cav.) Willd. & JIMT 42 B, 846, 881 & $\mathrm{Ab}$ & $\mathrm{Be}, \mathrm{Ms}$ & \\
\hline Desmodium orbiculare Schltdl. & JIMT 644, RPM 4881 & $\mathrm{He}$ & $\mathrm{Be}, \mathrm{Bg}, \mathrm{Ms}$ & MX \\
\hline Desmodium procumbens (Mill.) Hitchc. & JIMT 677, 726, & $\mathrm{He}$ & Bep, Bg & \\
\hline Desmodium psilophyllum Schltdl. & JIMT 662,721 & $\mathrm{He}$ & $\mathrm{Be}, \mathrm{Bg}$ & \\
\hline Desmodium sericophyllum Schltdl. & JIMT 548,722 & $\mathrm{He}$ & Bep, Bg & \\
\hline Erythrina coralloides DC. & JIMT 113, 413 & $\mathrm{Ar}$ & $\mathrm{Be}, \mathrm{Ms}$ & \\
\hline Erythrina leptorhiza DC. & JIMT 165, 995 & $\mathrm{He}$ & Be, Bep & MX \\
\hline Eysenhardtia polystachya (Ortega) Sarg. & JIMT 530 & $\mathrm{Ar}$ & Ms & \\
\hline Havardia pallens (Benth.) Britton \& Rose & JIMT 735, 950, RPM 5164, 5193 & $\mathrm{Ab}$ & Bep, Ms & \\
\hline Lathyrus parviflorus Roth & JIMT 517, 964 & $\operatorname{Tr}$ & $\mathrm{Be}$ & \\
\hline Lupinus marschallianus Sweet & JIMT 771 & $\mathrm{He}$ & Be, Bep & MX \\
\hline Lysiloma acapulcense (Kunth) Benth. & RPM 5168 & $\mathrm{Ar}$ & Bep & \\
\hline Lysiloma divaricatum (Jacq.) J.F.Macbr. & JIMT 107, 736, 947 & $\mathrm{Ar}$ & $\mathrm{Bg}, \mathrm{Ms}$ & \\
\hline Mariosousa mammifera (Schltdl.) Seigler \& Ebinger & JIMT 194, 446, PCL 1085 & $\mathrm{Ar}$ & $\mathrm{Bg}, \mathrm{Bm}$ & $\mathrm{MX}$ \\
\hline${ }^{1}$ Medicago lupulina $\mathrm{L}$. & JIMT 500, 963 & $\mathrm{He}$ & $\mathrm{Be}$ & \\
\hline Mimosa aculeaticarpa Ortega & JIMT 178 & $\mathrm{Ab}$ & Be, Bep & \\
\hline Mimosa albida Willd. & JIMT 699 & $\operatorname{Tr}$ & $\mathrm{Bg}, \mathrm{Ms}$ & \\
\hline Nissolia platycarpa Benth. & JIMT 636 & $\operatorname{Tr}$ & Ms & $\mathrm{MX}$ \\
\hline Painteria elachistophylla (S.Watson) Britton \& Rose & JIMT 122, 233, 373, 415, 627 & $\mathrm{Ab}$ & $\mathrm{Bep}, \mathrm{Bg}, \mathrm{Ms}$ & $\mathrm{MX}$ \\
\hline Phaseolus coccineus L. & JIMT 328, 787, RPM 5160 & $\operatorname{Tr}$ & Be, Bep, Bg & \\
\hline Phaseolus pedicellatus Benth. & JIMT 280, 599, 682, 786 & $\operatorname{Tr}$ & Be, Bep, Ms & \\
\hline Prosopis laevigata (Willd.) M.C.Johnst. & JIMT 493 & $\mathrm{Ar}$ & $\mathrm{Bg}$ & \\
\hline Senna crotalarioides (Kunth) H.S.Irwin \& Barneby & JIMT 634 & $\mathrm{Ab}$ & Ms & MX \\
\hline Senna hirsuta (L.) H.S.Irwin \& Barneby & JIMT 729 & $\mathrm{Ab}$ & Ms & \\
\hline Senna multiglandulosa (Jacq.) H.S.Irwin \& Barneby & JIMT 976 & $\mathrm{Ab}$ & $\mathrm{Be}$ & \\
\hline Senna septemtrionalis (Viv.) H.S.Irwin \& Barneby & JIMT 349, PCL 1111, RPM 5224 & $\mathrm{Ab}$ & $\mathrm{Be}, \mathrm{Bg}$ & \\
\hline
\end{tabular}


Flora de la microcuenca del Cañón de Los Chivos

\begin{tabular}{|c|c|c|c|c|}
\hline Clado/Familia/Especie & Recolector y no. de recolecta & F. de crec. y nutr. & T. de veg. & Endem. \\
\hline Trifolium amabile Kunth & JIMT 543, 663, 989 & $\mathrm{He}$ & Be, Bep & \\
\hline Vachellia farnesiana (L.) Wight \& Arn. & $\begin{array}{l}\text { RPM 5195, JIMT 42, 299, 481, } \\
960\end{array}$ & $\mathrm{Ab}$ & Bep, Bg, Ms & \\
\hline Vachellia pennatula (Schltdl. \& Cham.) Seigler \& Ebinger & JIMT 105 & $\mathrm{Ar}$ & Ms & \\
\hline \multicolumn{5}{|l|}{ Fagaceae } \\
\hline Quercus affinis Scheidw. & JIMT 384, RPM 5172 & $\mathrm{Ar}$ & Bep & MX \\
\hline Quercus castanea Née & JIMT 179,440 & $\mathrm{Ar}$ & Be, Bep & \\
\hline Quercus crassifolia Bonpl. & JIMT 365 & $\mathrm{Ar}$ & Bep & \\
\hline Quercus crassipes Bonpl. & JIMT 159, 183 & $\mathrm{Ar}$ & Be, Bep & MX \\
\hline Quercus furfuracea Liebm. & JIMT 418,370 & $\mathrm{Ar}$ & $\mathrm{Be}, \mathrm{Bg}, \mathrm{Bm}$ & MX \\
\hline Quercus jonesii Trel. & JIMT 180, 370 & $\mathrm{Ar}$ & Bep & MX \\
\hline Quercus laeta Liebm. & JIMT 168, RPM 5173 & $\mathrm{Ar}$ & $\mathrm{Be}, \mathrm{Bep}, \mathrm{Bg}$ & MX \\
\hline Quercus mexicana Bonpl. & JIMT 402, JIMT 978 & $\mathrm{Ar}$ & $\mathrm{Be}, \mathrm{Bg}$ & MX \\
\hline Quercus obtusata Bonpl. & JIMT 416 & $\mathrm{Ar}$ & $\mathrm{Be}, \mathrm{Bg}, \mathrm{Ms}$ & MX \\
\hline Quercus polymorpha Schltdl. \& Cham. & JIMT 88 & $\mathrm{Ar}$ & $\mathrm{Bg}, \mathrm{Bm}, \mathrm{Ms}$ & \\
\hline Quercus repanda Bonpl. & JIMT 368 & $\mathrm{Ab}$ & Bep & MX \\
\hline Quercus resinosa Liebm. & JIMT 181, RPM 4876 & $\mathrm{Ar}$ & Be, Bep & MX \\
\hline Quercus rugosa Née & JIMT 160 & $\mathrm{Ar}$ & $\mathrm{Be}$ & \\
\hline Quercus sapotifolia Liebm. & JIMT 130 & $\mathrm{Ar}$ & Bep, Bg & \\
\hline Quercus sebifera Trel. & JIMT 483 & $\mathrm{Ar}$ & $\mathrm{Be}$ & MX \\
\hline Quercus viminea Trel. & JIMT 182 & $\mathrm{Ar}$ & Bep & MX \\
\hline Quercus xalapensis Bonpl. & RPM 5174 & $\mathrm{Ar}$ & Bep & MX \\
\hline
\end{tabular}

\section{Garryaceae}

Garrya laurifolia Benth.

JIMT 513, RPM 5175

Ar

Be, Bep

\section{Geraniaceae}

Geranium seemannii Peyr.

JIMT 670, 816, RPM 4883, 5185

$\mathrm{He}$

Be, Bep

\section{Grossulariaceae}

Ribes affine Kunth

FGS 8493

$\mathrm{He}$

PCL 1095

$\operatorname{Tr}$

$\mathrm{Bg}$

Philadelphus mexicanus Schltdl.

JIMT 891

$\mathrm{He}$

Ms

Hypericum philonotis Schltdl. \& Cham.

Juglandaceae

Carya illinoinensis (Wangenh.) K.Koch

JIMT 246

Carya ovata (Mill.) K.Koch

RPM 5210, JIMT 404

Juglans mollis Engelm.

JIMT 100, 109, 485

\section{Lamiaceae}

Agastache palmeri (B.L. Rob.) Standl.

JIMT 764

Hedeoma drummondii Benth.

JIMT 531
$\mathrm{Ar}$

$\mathrm{Ar}$

$\mathrm{Ar}$

$\mathrm{He}$

$\mathrm{He}$

$$
\mathrm{Bg}
$$

$\mathrm{Be}, \mathrm{Bep}, \mathrm{Bg}, \mathrm{Bm}$

$\mathrm{Be}, \mathrm{Bg}, \mathrm{Ms} \quad \mathrm{MX}$

$\mathrm{Be}$

MX 
Morales et al. / Botanical Sciences 98(4): 644-681. 2020

\begin{tabular}{|c|c|c|c|c|}
\hline Clado/Familia/Especie & Recolector y no. de recolecta & F. de crec. y nutr. & T. de veg. & Endem. \\
\hline Hedeoma palmeri Hemsl. & JIMT 594, 669 & $\mathrm{He}$ & Be, Bep & MX \\
\hline Lepechinia caulescens (Ortega) Epling & JIMT 776 & $\mathrm{Ab}$ & $\mathrm{Be}$ & \\
\hline Salvia blepharophylla Brandegee ex Epling & RPM 5283 & $\mathrm{He}$ & Bep & MX \\
\hline Salvia mexicana $\mathrm{L}$. & JIMT 651,871 & $\mathrm{He}$ & $\mathrm{Be}, \mathrm{Bg}$ & MX \\
\hline Salvia microphylla Kunth & JIMT 190, 515, 925 & $\mathrm{He}$ & $\mathrm{Be}, \mathrm{Bg}, \mathrm{Ms}$ & \\
\hline Salvia patens Cav. & PCL 1112 & $\mathrm{He}$ & Bep, Bg & MX \\
\hline Salvia prunelloides Kunth & JIMT 566 & $\mathrm{He}$ & $\mathrm{Be}$ & MX \\
\hline Salvia puberula Fernald & $\begin{array}{l}\text { JIMT 769, RPM 4884, 5165, 5184, } \\
5209,5227\end{array}$ & $\mathrm{He}$ & Be, Bep & MX \\
\hline Scutellaria dumetorum Schltdl. & JIMT 619, 648, 794 & $\mathrm{He}$ & $\mathrm{Be}, \mathrm{Bg}$ & \\
\hline Scutellaria potosina Brandegee & PCL 1110 & $\mathrm{He}$ & $\mathrm{Be}, \mathrm{Bg}$ & \\
\hline Scutellaria seleriana Loes. & PCL 1092 & $\mathrm{He}$ & $\mathrm{Be}, \mathrm{Bg}$ & \\
\hline Stachys biflora Hook. \& Arn. & JIMT 785 & $\mathrm{He}$ & $\mathrm{Be}$ & \\
\hline Stachys coccinea Ortega & JIMT 297, 700, 806, PCL 1119 & $\mathrm{He}$ & $\mathrm{Be}, \mathrm{Bg}$ & \\
\hline Teucrium cubense Jacq. & JIMT 488, 730 & $\mathrm{He}$ & $\mathrm{Bg}, \mathrm{Ms}$ & \\
\hline \multicolumn{5}{|l|}{ Lentibulariaceae } \\
\hline Pinguicula ehlersiae Speta \& F. Fuchs & JIMT 2 & $\mathrm{He}$ & $\mathrm{Be}, \mathrm{Ms}$ & SLP \\
\hline Pinguicula macrophylla Kunth & JIMT 254 & $\mathrm{He}$ & $\mathrm{Bg}, \mathrm{Ms}$ & MX \\
\hline Pinguicula moranensis Kunth & $\begin{array}{l}\text { JIMT } 67,91,124,381, \text { RPM } 4787 \text {, } \\
5289\end{array}$ & $\mathrm{He}$ & $\mathrm{Be}, \mathrm{Bep}, \mathrm{Bg}, \mathrm{Bm}$ & \\
\hline \multicolumn{5}{|l|}{ Loasaceae } \\
\hline Mentzelia hispida Willd. & JIMT 304 & $\mathrm{Ab}$ & Ms & \\
\hline \multicolumn{5}{|l|}{ Lythraceae } \\
\hline Cuphea aequipetala Cav. & JIMT 235, RPM 5178 & $\mathrm{He}$ & Bep, Ms & \\
\hline Lythrum gracile Benth. & JIMT 668 & $\mathrm{He}$ & $\mathrm{Be}$ & \\
\hline \multicolumn{5}{|l|}{ Malpighiaceae } \\
\hline Callaeum septentrionale (A. Juss.) D.M.Johnson & RPM 5180, JIMT 33, 279 & $\operatorname{Tr}$ & Bep, Ms & MX \\
\hline \multicolumn{5}{|l|}{ Malvaceae } \\
\hline Herissantia crispa (L.) Brizicky & JIMT 941 & $\mathrm{He}$ & Ms & \\
\hline Phymosia umbellata (Cav.) Kearney & JIMT 37 & $\operatorname{Ar}$ & $\mathrm{Be}, \mathrm{Ms}$ & \\
\hline Sida abutifolia Mill. & JIMT 753, 948 & $\mathrm{He}$ & Ms & \\
\hline Sida acuta Burm.f. & JIMT 766, 795 & $\mathrm{He}$ & $\mathrm{Be}$ & \\
\hline Sida haenkeana C.Presl & JIMT 733, 920 & $\mathrm{He}$ & $\mathrm{Bg}, \mathrm{Ms}$ & \\
\hline Sida rhombifolia L. & JIMT 240, 516, 499, 958 & $\mathrm{He}$ & $\mathrm{Be}, \mathrm{Ms}$ & \\
\hline Tilia americana subsp. mexicana (Schltdl.) Hardin & JIMT 193, 1000 & $\operatorname{Ar}$ & $\mathrm{Be}, \mathrm{Bg}, \mathrm{Bm}$ & \\
\hline \multicolumn{5}{|l|}{ Moraceae } \\
\hline Ficus cotinifolia Kunth & JIMT 1, 489 & $\operatorname{Ar}$ & $\mathrm{Bg}, \mathrm{Bm}, \mathrm{Ms}$ & \\
\hline Morus celtidifolia Kunth & JIMT 106 & $\mathrm{Ar}$ & $\mathrm{Bg}, \mathrm{Bm}$ & \\
\hline \multicolumn{5}{|l|}{ Myricaceae } \\
\hline Morella cerifera (L.) Small & JIMT 133, 393 & $\mathrm{Ab}$ & Bep, Bg & \\
\hline
\end{tabular}


Clado/Familia/Especie

Nyctaginaceae

Mirabilis jalapa L.

Oleaceae

Forestiera reticulata Torr.

Fraxinus uhdei (Wenz.) Lingelsh.

\section{Onagraceae}

Oenothera kunthiana (Spach) Munz

Oenothera tetraptera Cav.

\section{Orobanchaceae}

Castilleja integrifolia L.f.

Castilleja mexicana (Hemsl.) A.Gray

Castilleja scorzonerifolia Kunth

Conopholis alpina Liebm.

Lamourouxia rhinanthifolia Kunth

Seymeria virgata (Kunth) Benth.

\section{Oxalidaceae}

Oxalis decaphylla Kunth

Oxalis latifolia Kunth

\section{Papaveraceae}

Argemone mexicana $\mathrm{L}$.

\section{Passifloraceae}

Passiflora subpeltata Ortega

Phyllanthaceae

Phyllanthus barbarae M.C.Johnst.

\section{Phytolaccaceae}

Phytolacca icosandra L.

Rivina humilis L.

\section{Plantaginaceae}

Maurandya antirrhiniflora Humb. \& Bonpl. ex Willd.

Nuttallanthus canadensis (L.) D.A.Sutton

Penstemon barbatus (Cav.) Roth

Plantago australis Lam.

Plantago nivea Kunth

Russelia polyedra Zucc.

\section{Platanaceae}

Platanus mexicana Moric.

\section{Plumbaginaceae}

Plumbago pulchella Boiss.

Plumbago zeylanica L.

JIMT 593
JIMT 926

PCL 1097

PCL 1103, RPM 5550

$\mathrm{Ab}$

$\mathrm{Be}, \mathrm{Ms}$

JIMT $85,428,893$

JIMT 86 B

Ar

$\mathrm{Ar}$

$\mathrm{Be}, \mathrm{Bg}, \mathrm{Bm}$

JIMT 497, 813

$\mathrm{He}$

$\mathrm{He}$

$\mathrm{Be}$

JIMT 401, 929

JIMT 888

JIMT 849

JIMT 420, 498, 580, 760, 789, 825

JIMT 375, RPM 5218

JIMT 305

JIMT 595

JIMT 540, 683, RPM 4869

JIMT 552, 586

JIMT 490

JIMT 111, 447, RPM 5200, 5553

JIMT 613

JIMT 752

JIMT 928

JIMT 492

JIMT 557, 817

JIMT 234

JIMT 184, 405, RPM 5522

JIMT 307, 955

JIMT 306, 473, 701

$\mathrm{Bg}, \mathrm{Ms}$
$\mathrm{He}$

$\mathrm{He}$

$\mathrm{He}$

$\operatorname{Tr}$

$\mathrm{Be}, \mathrm{Bg}, \mathrm{Bm}, \mathrm{Ms}$

$\mathrm{Ab}$

$\mathrm{He}$

$\mathrm{Ab}$

Bep, Bg

$\mathrm{He}, \mathrm{Pa}$

$\mathrm{He}, \mathrm{Pa}$

$\mathrm{He}, \mathrm{Pa}$

Be, Bep

Be, Bep

Ms

Bep

MX

MX

Be, Bep, Ms

Bep

$\mathrm{Bg}$

Ms

$\operatorname{Tr}$

$\mathrm{Be}, \mathrm{Bg}$

$\mathrm{He}$

$\mathrm{Bg}$

$\mathrm{He}$

Bep

$\mathrm{He}$

$\mathrm{Be}, \mathrm{Bg}$

$\mathrm{He}$

Bep

$\mathrm{He}$

Ms

MX

Ar

$\mathrm{Bg}$

$\mathrm{Ab}$

Ms

MX 
Morales et al. / Botanical Sciences 98(4): 644-681. 2020

\begin{tabular}{|c|c|c|c|c|}
\hline Clado/Familia/Especie & Recolector y no. de recolecta & F. de crec. y nutr. & T. de veg. & Endem. \\
\hline \multicolumn{5}{|l|}{ Polemoniaceae } \\
\hline Loeselia coerulea (Cav.) G. Don & JIMT 853,938, & $\mathrm{He}$ & Ms & \\
\hline Loeselia mexicana (Lam.) Brand & JIMT 908 & $\mathrm{He}$ & Bep & \\
\hline \multicolumn{5}{|l|}{ Polygalaceae } \\
\hline Polygala barbeyana Chodat & JIMT 611 & $\mathrm{He}$ & $\mathrm{Bg}$ & \\
\hline Polygala buxifolia Kunth & JIMT 996 B & $\mathrm{He}$ & Bep & MX \\
\hline Persicaria punctata (Elliott) Small & JIMT 724, 916 & $\mathrm{He}$ & $\mathrm{Bg}$ & \\
\hline${ }^{1}$ Rumex obtusifolius L. & RPM 5226 & $\mathrm{He}$ & $\mathrm{Be}$ & \\
\hline \multicolumn{5}{|l|}{ Primulaceae } \\
\hline${ }^{1}$ Anagallis arvensis $\mathrm{L}$. & JIMT 671 & $\mathrm{He}$ & $\mathrm{Be}$ & \\
\hline \multicolumn{5}{|l|}{ Ranunculaceae } \\
\hline Clematis dioica $\mathrm{L}$. & PCL 1104 & $\operatorname{Tr}$ & $\mathrm{Bg}$ & \\
\hline Clematis pitcheri Torr. \& A.Gray & JIMT 230, 654, RPM 4857 & $\operatorname{Tr}$ & $\mathrm{Be}$ & \\
\hline Clematis rhodocarpa Rose & JIMT 807 & $\operatorname{Tr}$ & $\mathrm{Be}$ & MX \\
\hline Ranunculus petiolaris Kunth & JIMT $167,579,717$ & $\mathrm{He}$ & $\mathrm{Be}, \mathrm{Bep}, \mathrm{Bg}$ & \\
\hline Thalictrum strigillosum Hemsl. & JIMT 205, 612 & $\mathrm{He}$ & $\mathrm{Be}, \mathrm{Bg}$ & MX \\
\hline \multicolumn{5}{|l|}{ Rhamnaceae } \\
\hline Ceanothus caeruleus Lag. & JIMT 696, 902 & Ar & $\mathrm{Bep}, \mathrm{Bg}$ & \\
\hline Colubrina ehrenbergii Schltdl. & JIMT 632,892 & $\mathrm{Ar}$ & Ms & MX \\
\hline Colubrina greggii S.Watson & $\begin{array}{l}\text { JIMT 534, 741, PCL 1086, RPM } \\
5163\end{array}$ & $\mathrm{Ar}$ & $\mathrm{Bg}, \mathrm{Bm}, \mathrm{Ms}$ & \\
\hline $\begin{array}{l}\text { Frangula microphylla (Humb. \& Bonpl. ex Schult.) } \\
\text { Grubov }\end{array}$ & JIMT 576 & $\mathrm{Ab}$ & Bep & MX \\
\hline Karwinskia mollis Schltdl. & JIMT 335 & $\mathrm{Ab}$ & $\mathrm{Be}, \mathrm{Ms}$ & MX \\
\hline Rhamnus longistyla C.B. Wolf & JIMT 157 & $\mathrm{Ar}$ & $\mathrm{Be}$ & \\
\hline Rhamnus serrata Willd. ex Schult. & JIMT 808 & $\mathrm{Ar}$ & $\mathrm{Be}$ & $\mathrm{MX}$ \\
\hline \multicolumn{5}{|l|}{ Rosaceae } \\
\hline Crataegus rosei Eggl. & JIMT 112 & $\mathrm{Ar}$ & $\mathrm{Be}, \mathrm{Bg}$ & MX \\
\hline Malacomeles denticulata (Kunth) G.N.Jones & JIMT 527, 894 & $\mathrm{Ar}$ & $\mathrm{Be}, \mathrm{Ms}$ & \\
\hline Prunus rhamnoides Koehne & JIMT 129, 449 & $\mathrm{Ar}$ & $\mathrm{Bep}, \mathrm{Bg}, \mathrm{Bm}$ & \\
\hline Prunus serotina Ehrh. & JIMT 118 & $\mathrm{Ar}$ & Bep & \\
\hline Rubus pringlei $\mathrm{Rydb}$. & JIMT 117, 390 & $\operatorname{Tr}$ & Be, Bep & \\
\hline Vauquelinia corymbosa Corr^a ex Humb. \& Bonpl. & JIMT 672, RPM 5191 & $\mathrm{Ar}$ & $\mathrm{Be}, \mathrm{Ms}$ & \\
\hline \multicolumn{5}{|l|}{ Rubiaceae } \\
\hline Bouvardia multiflora (Cav.) Schult. & JIMT 231 & $\mathrm{He}$ & $\mathrm{Be}, \mathrm{Bg}$ & \\
\hline Bouvardia ternifolia (Cav.) Schltdl. & RPM 4865, JIMT 193 B & $\mathrm{He}$ & $\mathrm{Be}, \mathrm{Bg}$ & \\
\hline Chiococca pachyphylla Wernham & JIMT 31, 431, 867 & $\mathrm{Ar}$ & $\mathrm{Be}, \mathrm{Bm}$ & \\
\hline Chione venosa (Sw.) Urb. & PCL 1094 & $\operatorname{Ar}$ & $\mathrm{Bg}, \mathrm{Bm}$, & \\
\hline Deppea cornifolia (Benth.) Benth. & RPM 4790, 5192, 5208 & $\mathrm{Ab}$ & Be, Bep & MX \\
\hline Galium uncinulatum DC. & JIMT 660 & $\mathrm{He}$ & $\mathrm{Be}$ & \\
\hline
\end{tabular}


Flora de la microcuenca del Cañón de Los Chivos

\begin{tabular}{|c|c|c|c|c|}
\hline Clado/Familia/Especie & Recolector y no. de recolecta & F. de crec. y nutr. & T. de veg. & Endem. \\
\hline Randia capitata DC. & JIMT 98, RPM 5170 & $\mathrm{Ab}$ & Bep, Ms & \\
\hline Spermacoce verticillata $\mathrm{L}$. & JIMT 549 & $\mathrm{He}$ & Bep & \\
\hline Stenaria nigricans (Lam.) Terrell & JIMT 610 & $\mathrm{He}$ & $\mathrm{Bg}$ & \\
\hline \multicolumn{5}{|l|}{ Rutaceae } \\
\hline Casimiroa edulis La Llave & JIMT 101 & $\mathrm{Ar}$ & Ms & \\
\hline Decatropis bicolor (Zucc.) Radlk. & JIMT 200, RPM,5171 & $\mathrm{Ar}$ & $\mathrm{Be}, \mathrm{Ms}$ & MX \\
\hline Esenbeckia berlandieri Baill. & JIMT 10, JIMT 21, RPM 5161 & $\mathrm{Ar}$ & $\mathrm{Bm}, \mathrm{Ms}$ & \\
\hline Ptelea trifoliata $\mathrm{L}$. & JIMT 271 & $\mathrm{Ar}$ & Ms & \\
\hline \multicolumn{5}{|l|}{ Salicaceae } \\
\hline Salix humboldtiana Willd. & JIMT 445 & $\mathrm{Ar}$ & $\mathrm{Bg}$ & \\
\hline Salix taxifolia Kunth & JIMT 255 & $\mathrm{Ab}$ & $\mathrm{Bg}$ & \\
\hline \multicolumn{5}{|l|}{ Santalaceae } \\
\hline Phoradendron bolleanum (Seem.) Eichler & JIMT 855 & Ep, Pa & Bep, Ms & \\
\hline Phoradendron longifolium Eichler ex Trel. & JIMT 57, 82, 687, 882, 906 & Ep, Pa & $\mathrm{Be}, \mathrm{Bep}, \mathrm{Bg}, \mathrm{Ms}$ & MX \\
\hline Phoradendron velutinum (DC.) Oliv. & JIMT 972 & $\mathrm{Ep}, \mathrm{Pa}$ & $\mathrm{Be}$ & \\
\hline \multicolumn{5}{|l|}{ Sapindaceae } \\
\hline Dodonaea viscosa (L.) Jacq. & JIMT 43 B, JIMT 933, RPM 4878 & $\mathrm{Ab}$ & $\mathrm{Be}, \mathrm{Bep}, \mathrm{Ms}$ & \\
\hline Paullinia tomentosa Jacq. & JIMT 639 & $\operatorname{Tr}$ & Ms & \\
\hline \multicolumn{5}{|l|}{ Saxifragaceae } \\
\hline Heuchera mexicana W. Schaffn. ex Rydb. & JIMT 987 & $\mathrm{He}$ & Be, Bep & MX \\
\hline \multicolumn{5}{|l|}{ Scrophulariaceae } \\
\hline Buddleja cordata Kunth & RPM 5230; PCL 1143JIMT 448 & $\mathrm{Ar}$ & $\mathrm{Be}, \mathrm{Bep}, \mathrm{Bg}$ & \\
\hline Buddleja scordioides Kunth & JIMT 935 & $\mathrm{Ab}$ & $\mathrm{Bg}, \mathrm{Ms}$ & \\
\hline \multicolumn{5}{|l|}{ Solanaceae } \\
\hline Capsicum annuum $\mathrm{L}$. & JIMT 30 & $\mathrm{Ab}$ & Ms & \\
\hline Capsicum rhomboideum (Dunal) Kuntze & PCL 1077 & $\mathrm{Ab}$ & $\mathrm{Bg}, \mathrm{Bm}$ & \\
\hline Cestrum oblongifolium Schltdl. & $\begin{array}{l}\text { JIMT 46, 395, 408, 781, 876, 905, } \\
\text { 966, RPM } 5231\end{array}$ & $\mathrm{Ab}$ & Be, Bep, Bg & MX \\
\hline Datura stramonium L. & JIMT 298 & $\mathrm{He}$ & Bep, Bg, Ms & \\
\hline Lycianthes heteroclita (Sendtn.) Bitter & PCL 1078 & $\mathrm{He}$ & $\mathrm{Bg}$ & \\
\hline${ }^{1}$ Nicotiana glauca Graham & JIMT 963 & $\mathrm{He}$ & $\mathrm{Bg}$ & \\
\hline${ }^{1}$ Nicotiana tabacum L. & JIMT 432 & $\mathrm{He}$ & Bep & \\
\hline Physalis chenopodifolia Lam. & JIMT 589 & $\mathrm{He}$ & Be, Bep & MX \\
\hline Physalis coztomatl Dunal & JIMT 777 & $\mathrm{He}$ & $\mathrm{Be}$ & MX \\
\hline Physalis patula Mill. & JIMT 945 & $\mathrm{He}$ & Ms & MX \\
\hline Solanum erianthum D. Don & JIMT 192, 450, 740, 940 & $\mathrm{Ar}$ & $\mathrm{Bg}, \mathrm{Bm}, \mathrm{Ms}$ & \\
\hline${ }^{1}$ Solanum marginatum L. f. & JIMT 80, 915 & $\mathrm{He}$ & Bep, bg, Ms & \\
\hline Solanum nigrescens $\mathrm{M}$. Martens \& Galeotti & JIMT 797 & $\mathrm{He}$ & $\mathrm{Be}$ & \\
\hline${ }^{1}$ Solanum physalifolium Rusby & JIMT 692 & $\mathrm{He}$ & $\mathrm{Bg}$ & \\
\hline
\end{tabular}


Morales et al. / Botanical Sciences 98(4): 644-681. 2020

\begin{tabular}{|c|c|c|c|c|}
\hline Clado/Familia/Especie & Recolector y no. de recolecta & F. de crec. y nutr. & T. de veg. & Endem. \\
\hline \multicolumn{5}{|l|}{ Staphyleaceae } \\
\hline Staphylea pringlei $\mathrm{S}$. Watson & JIMT 3, 83, 443 & $\mathrm{Ar}$ & $\mathrm{Bg}, \mathrm{Bm}$ & MX \\
\hline \multicolumn{5}{|l|}{ Talinaceae } \\
\hline Talinum paniculatum (Jacq.) Gaertn. & JIMT 743 & $\mathrm{He}$ & $\mathrm{Bg}, \mathrm{Ms}$ & \\
\hline \multicolumn{5}{|l|}{ Urticaceae } \\
\hline Pilea microphylla (L.) Liebm. & JIMT 273, 344, 539; RPM 4793 & $\mathrm{He}$ & $\mathrm{Be}, \mathrm{Bg}, \mathrm{Ms}$ & \\
\hline \multicolumn{5}{|l|}{ Verbenaceae } \\
\hline Citharexylum oleinum (Benth. ex Lindl.) Moldenke & JIMT 202, 425 & $\mathrm{Ab}$ & $\mathrm{Be}, \mathrm{Bg}$ & MX \\
\hline Glandularia elegans (Kunth) Umber & $\begin{array}{l}\text { JIMT 41, 433, 847, 909, 965; RPM } \\
5235\end{array}$ & $\mathrm{He}$ & $\mathrm{Be}, \mathrm{Bep}, \mathrm{Ms}$ & \\
\hline Lantana camara L. & JIMT 268, 758, 939 & $\mathrm{Ab}$ & Ms & \\
\hline Lantana canescens Kunth & JIMT 884 & $\mathrm{Ab}$ & Ms & \\
\hline Verbena carolina $\mathrm{L}$. & JIMT 746 & $\mathrm{He}$ & Ms & \\
\hline Verbena menthifolia Benth. & JIMT 649, 791 & $\mathrm{He}$ & $\mathrm{Be}$ & \\
\hline \multicolumn{5}{|l|}{ Violaceae } \\
\hline Viola hookeriana Kunth & JIMT 792, 967 & $\mathrm{He}$ & Be, Bep, Bg & \\
\hline \multicolumn{5}{|l|}{ Vitaceae } \\
\hline Parthenocissus quinquefolia (L.) Planch. & JIMT 999 & $\operatorname{Tr}$ & Be, Bep, Bg & \\
\hline Vitis tiliifolia Humb. \& Bonpl. ex Schult. & JIMT 774 & $\operatorname{Tr}$ & Be, Bep, Bg, Bm & \\
\hline
\end{tabular}


Apéndice 2. Especies dentro de la NOM-059: Amenazada (A), en peligro de extinción (P), protección especial (Pr). CITES: apéndice I (I), apéndice II (II). IUCN: en peligro crítico (CR), en peligro (EN), vulnerable (VU), próxima a la vulnerabilidad (NT), preocupación menor (LC), dependiente de medidas de conservación (LR/CD) de acuerdo con la antigua categoría (Lower risk: conservation dependant).

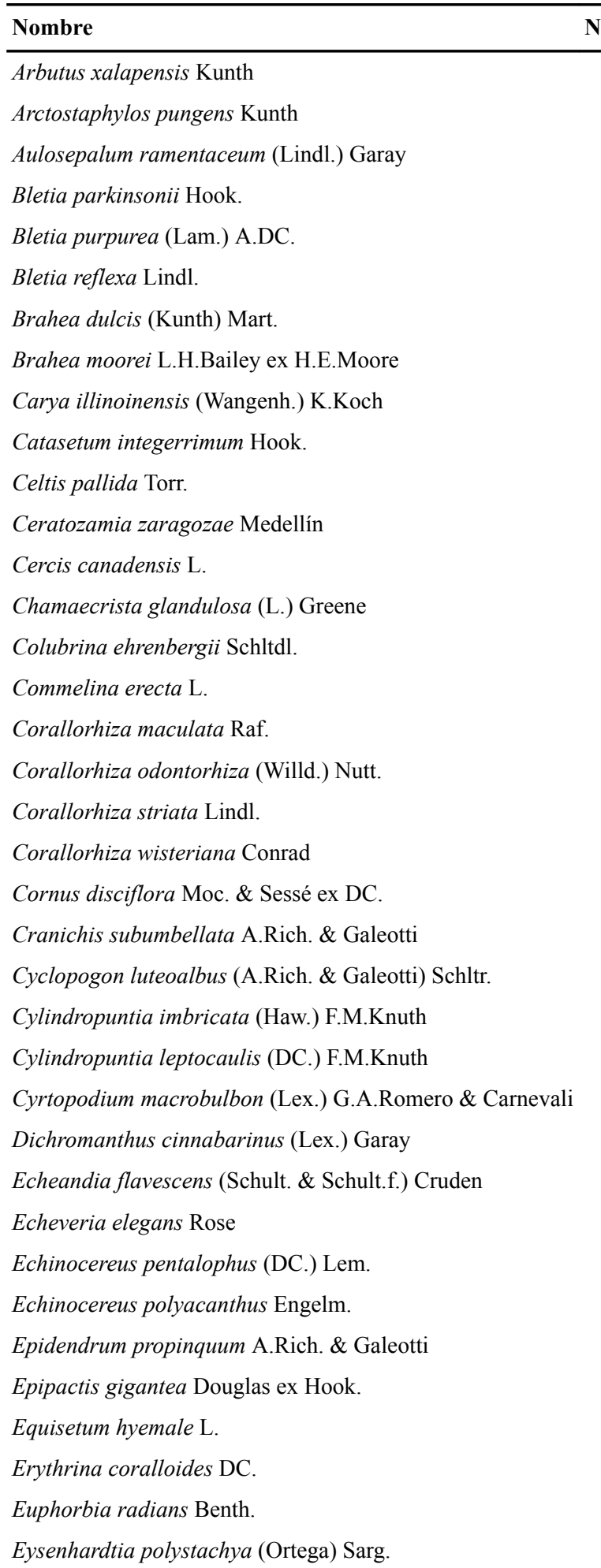

Nom-059 CITES IUCN

$\operatorname{Pr}$

LC

Pr LC

LC

II

LC

P $\quad$ I $\quad$ CR

LC

LC

LC

LC

II

II

II

II

VU

II

II

II

LC

II

II

II

LC

P

II LC

II LC

II

II LC

LC

A

II

LC 
Morales et al. / Botanical Sciences 98(4): 644-681. 2020

Nombre

Ferocactus echidne (DC.) Britton \& Rose

Ferocactus glaucescens (DC.) Britton \& Rose

Ferocactus histrix (DC.) G.E.Linds.

Ficus cotinifolia Kunth

Fraxinus uhdei (Wenz.) Lingelsh.

Funkiella tenella (L.O.Williams) Szlach.

Gaultheria erecta Vent.

Govenia lagenophora Lindl.

Govenia liliacea (Lex.) Lindl.

Govenia purpusii Schltr.

Havardia pallens (Benth.) Britton \& Rose

Hexalectris grandiflora (A.Rich. \& Galeotti) L.O.Williams

Hexalectris nitida L.O.Williams

Ilex brandegeeana Loes.

Juncus acuminatus Michx.

Juniperus flaccida Schltdl.

Laelia anceps Lindl.

Litsea glaucescens Kunth

Lysiloma acapulcense (Kunth) Benth.

Lysiloma divaricatum (Jacq.) J.F.Macbr.

Malaxis brachystachys Rchb.f.

Malaxis macrostachya (Lex.) Kuntze

Mammillaria densispina (J.M. Coult.) Orcutt

Mammillaria erythrosperma Boed.

Mammillaria schiedeana Ehrenb. ex Schltdl.

Mammillaria uncinata Zucc. ex Pfeiff.

Mesadenus polyanthus (Rchb.f.) Schltr.

Mimosa aculeaticarpa Ortega

Myrtillocactus geometrizans (Mart. ex Pfeiff.) Console

Opuntia leucotricha DC.

Opuntia megarrhiza Rose

Opuntia neochrysacantha Bravo

Opuntia pachyrrhiza H.M. Hern., Gomez-Hin. \& Barcenas

Opuntia robusta J.C. Wendl.

Pennisetum glaucum (L.) R.Br.

Persea americana Mill.

Persea liebmannii Mez

Physalis chenopodiifolia Willd.

Physalis coztomatl Dunal

Physalis patula Mill.

Pinus arizonica Engelm.

Pinus cembroides Zucc.
Nom-059 CITES IUCN

$\begin{array}{ccc} & \text { II } & \text { LC } \\ & \text { II } & \text { LC } \\ & \text { II } & \text { NT } \\ & & \text { LC } \\ & \text { LC } \\ \text { II } & \\ & \text { LC } \\ \text { II } & \\ \text { II } & \\ & \\ & \text { LC } \\ \text { II } & \\ \text { II } & \\ & \text { LC } \\ & \text { LC }\end{array}$

A

$\mathrm{LC}$

LC

II LC

II

II LC

A II LC

A II VU

II LC

II

LC

II LC

II LC

II EN

II

EN

II LC

LC

LC

LC

LC

LC

LC

LC

LC 
Nombre

Nom-059 CITES IUCN

Pinus devoniana Lindl.

LC

Pinus greggii Engelm. ex Parl.

VU

Pinus montezumae Lamb.

LC

Pinus pseudostrobus Lindl.

LC

Pinus strobiformis Engelm.

$\operatorname{Pr}$

LC

Pinus teocote Schied. ex Schltdl. \& Cham.

LC

Pistacia mexicana Kunth

NT

Poa аппиа L.

LC

Prosopis laevigata (Willd.) M.C.Johnst.

$\mathrm{LC}$

Prunus serotina Ehrh.

LC

Quercus affinis Scheidw.

LC

Quercus castanea Née

LC

Quercus crassifolia Bonpl.

$\mathrm{LC}$

Quercus crassipes Bonpl.

LC

Quercus jonesii Trel.

LC

Quercus laeta Liebm.

LC

Quercus mexicana Bonpl.

LC

Quercus obtusata Bonpl.

LC

Quercus polymorpha Schltdl. \& Cham.

LC

Quercus repanda Bonpl.

LC

Quercus resinosa Liebm.

LC

Quercus sapotifolia Liebm.

LC

Quercus sebifera Trel.

LC

Quercus viminea Trel.

$\mathrm{LC}$

Quercus xalapensis Bonpl.

LC

Rhamnus serrata Willd. ex Schult.

LC

Salix humboldtiana Willd.

LC

Salix taxifolia Kunth

LC

Schiedeella nagelii (L.O.Williams) Garay

$\operatorname{Pr}$

Sebastiania pavoniana (Müll.Arg.) Müll.Arg.

LC

Selenicereus spinulosus (DC.) Britton \& Rose

LC

Senna multiglandulosa (Jacq.) H.S.Irwin \& Barneby

LC

Sotoa confusa (Garay) Salazar

II

Stenocereus queretaroensis (F.A.C.Weber ex Mathes.)

Buxb.

II LC

Tilia americana var. mexicana (Schltdl.) Hardin

$\mathrm{P}$

Trifolium amabile Kunth

LC

Triphora trianthophora (Sw.) Rydb.

II

Viburnum elatum Benth.

$\mathrm{CD}$ 